\title{
Existence for a Second-Order Impulsive Neutral Stochastic Integrodifferential Equations with Nonlocal Conditions and Infinite Delay
}

\author{
Dang Huan Diem ${ }^{1,2}$ \\ ${ }^{1}$ Institute of Mathematics, School of Mathematical Science, Nanjing Normal University, \\ Nanjing 210023, China \\ ${ }^{2}$ Faculty of Basic Sciences, Bacgiang Agriculture and Forestry University, \\ Bacgiang 21000, Vietnam
}

Correspondence should be addressed to Dang Huan Diem; diemdanghuan80@gmail.com

Received 16 November 2013; Accepted 24 December 2013; Published 27 February 2014

Academic Editors: H. Xu and S. Zhang

Copyright (C) 2014 Dang Huan Diem. This is an open access article distributed under the Creative Commons Attribution License, which permits unrestricted use, distribution, and reproduction in any medium, provided the original work is properly cited.

\begin{abstract}
The current paper is concerned with the existence of mild solutions for a class of second-order impulsive neutral stochastic integrodifferential equations with nonlocal conditions and infinite delays in a Hilbert space. A sufficient condition for the existence results is obtained by using the Krasnoselskii-Schaefer-type fixed point theorem combined with theories of a strongly continuous cosine family of bounded linear operators. Finally, an application to the stochastic nonlinear wave equation with infinite delay is given.
\end{abstract}

\section{Introduction}

The theory of impulsive neutral differential equations has been emerging as an important area of investigation in recent years, stimulated by their numerous applications to problems from physics, mechanics, electrical engineering, medicine biology, ecology, and so on. Ordinary differential equations of first and second order with impulses have been treated in several works and we refer the reader to the monographs of Lakshmikantham et al. [1], the papers [2-5], and the references therein related to this matter. Besides, noise or stochastic perturbation is unavoidable and omnipresent in nature as well as in man-made systems. Therefore, it is of great significance to import the stochastic effects into the investigation of impulsive neutral differential equations. As the generalization of classic impulsive neutral differential equations, impulsive neutral stochastic integrodifferential equations with infinite delays have attracted the researchers' great interest. There are few publications on well-posedness of solutions for these equations (e.g., see, [6-8] and the references therein).
Recently, in [9], Cui and Yan proved sufficient conditions for the existence of fractional neutral stochastic integrodifferential equations with infinite delay of the form

$$
\begin{gathered}
D_{t}^{\alpha}\left[x(t)+G\left(t, x_{t}\right)\right] \\
=-A x(t)+f\left(t, x_{t}\right)+\int_{-\infty}^{t} \sigma\left(t, s, x_{s}\right) d W(s), \\
J:=[0, b], \\
x(t)=\phi(t), \quad t \in(-\infty, 0],
\end{gathered}
$$

where $0<\alpha<1$ and $D_{t}^{\alpha}$ denotes the Caputo fractional derivative operator of order $\alpha$ by means of Sadovskii's fixed point theorem. And very recently, also thanks to the Sadovskii fixed point theorem combined with a noncompact condition on the cosine family of operators, Arthi and Balachandran [10] established the controllability of the following damped 
second-order impulsive neutral functional differential systems with infinite delay:

$$
\begin{gathered}
\frac{d}{d t}\left[x^{\prime}(t)-g\left(t, x_{t}\right)\right]=A x(t)+\mathscr{D} x^{\prime}(t)+B u(t)+f\left(t, x_{t}\right), \\
t_{i} \neq t \in J:=[0, a], \\
x_{0}=\varphi \in \mathscr{B}, \quad x^{\prime}(0)=\xi \in X, \\
\Delta x\left(t_{i}\right)=I_{i}\left(x_{t_{i}}\right), \quad i=1, \ldots, n, \\
\Delta x^{\prime}\left(t_{i}\right)=J_{i}\left(x_{t_{i}}\right), \quad i=1, \ldots, n,
\end{gathered}
$$

where $\mathscr{D}$ is a bounded linear operator on a Banach space $X$ with $D(\mathscr{D}) \subset D(A)$.

On the other hand, there has not been very much study of second-order impulsive neutral stochastic functional differential equations with infinite delays, while these have begun to gain attention recently. To be more precise, in [11], Balasubramaniam and Muthukumar discussed on approximate controllability of second-order stochastic distributed implicit functional differential systems with infinite delay. Cui and Yan [12] investigated the existence of mild solutions for impulsive neutral second-order stochastic evolution equations with nonlocal conditions. Mahmudov and McKibben [13] established the results concerning the global existence, uniqueness, approximation, and exact controllability of mild solutions for a class of abstract second-order damped McKean-Vlasov stochastic evolution equations in a real separable Hilbert space. However, up to now, the well-posedness of mild solutions for a class of second-order impulsive neutral stochastic integro-differential equations with nonlocal conditions and infinite delays in a Hilbert spaces has not been considered in the literature. In order to fill this gap, based on ideas and techniques in the above works, in this paper, we will study the well-posedness of mild solutions for a class of second-order impulsive neutral stochastic integrodifferential equations with nonlocal conditions and infinite delays of the form

$$
\begin{gathered}
d\left[x^{\prime}(t)-g\left(t, x_{t}, \int_{0}^{t} \sigma_{1}\left(t, s, x_{s}\right) d s\right)\right] \\
=\left[A x(t)+f\left(t, x_{t}, \int_{0}^{t} \sigma_{2}\left(t, s, x_{s}\right) d s\right)\right] d t \\
\quad+\int_{-\infty}^{t} \sigma\left(t, s, x_{s}\right) d w(s), \quad t_{k} \neq t \in J:=[0, T], \\
\Delta x\left(t_{k}\right)=I_{k}^{1}\left(x\left(t_{k}^{-}\right)\right), \quad k=\{1, \ldots, m\}=: \overline{1, m}, \\
\Delta x^{\prime}\left(t_{k}\right)=I_{k}^{2}\left(x\left(t_{k}^{-}\right)\right), \quad k=\overline{1, m}, \\
x^{\prime}(0)=x_{1} \in \mathbb{U}, \\
x(0)-q\left(x_{t_{1}}, x_{t_{2}}, \ldots, x_{t_{n}}\right)=x_{0}=\varphi \in \mathscr{B}, \\
\text { for a.e. } s \in J_{0}:=(-\infty, 0] .
\end{gathered}
$$

Here, $x(\cdot)$ is a stochastic process taking values in a real

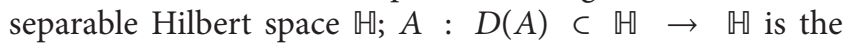
infinitesimal generator of a strongly continuous cosine family on $\mathbb{R}$. The history $x_{t}: J_{0} \rightarrow \mathbb{U}, x_{t}(\theta)=x(t+\theta)$ for $t \geq 0$, belongs to the phase space $\mathscr{B}$, which will be described in

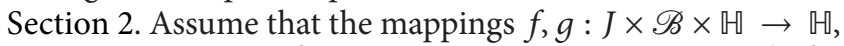
$\sigma: J \times J \times \mathscr{B} \rightarrow \mathscr{L}_{2}^{0}, \sigma_{i}: J \times J \times \mathscr{B} \rightarrow \mathbb{Q}, i=1,2, I_{k}^{1}, I_{k}^{2}:$ $\mathbb{\sharp} \rightarrow \mathbb{U}, k=\overline{1, m}, q: \mathscr{B}^{n} \rightarrow \mathscr{B}$ are appropriate functions to be specified later. Furthermore, let $0<t_{1}<\cdots<t_{m}<T$ be prefixed points, and $\Delta x\left(t_{k}\right)=x\left(t_{k}^{+}\right)-x\left(t_{k}^{-}\right)$represents the jump of the function $x$ at time $t_{k}$ with $I_{k}$ determining the size of the jump, where $x\left(t_{k}^{+}\right)$and $x\left(t_{k}^{-}\right)$represent the right and left limits of $x(t)$ at $t=t_{k}$, respectively. Similarly $x^{\prime}\left(t_{k}^{+}\right)$and $x^{\prime}\left(t_{k}^{-}\right)$ denote, respectively, the right and left limits of $x^{\prime}(t)$ at $t_{k}$. Let $\varphi(t) \in \mathscr{L}_{2}(\Omega, \mathscr{B})$ and $x_{1}(t)$ be $\mathbb{H}$-valued $\mathscr{F}_{t}$-measurable random variables independent of the Wiener process $W$ with a finite second moment.

The structure of this paper is as follows. In Section 2, we briefly present some basic notations, preliminaries, and assumptions. The main results in Section 3 are devoted to study the well-posedness of mild solutions for (3) with their proofs. An example is given in Section 4 to illustrate the theory. In the last section, concluding remarks are given.

\section{Preliminaries}

In this section, we briefly recall some basic definitions and results for stochastic equations in infinite dimensions and cosine families of operators. For more details on this section, we refer the reader to [14-16].

Let $\left(\mathbb{G},\|\cdot\|_{\mathbb{W}},\langle\cdot, \cdot\rangle_{\mathbb{W}}\right)$ and $\left(\mathbb{K},\|\cdot\|_{\mathbb{K}},\langle\cdot, \cdot\rangle_{\mathbb{K}}\right)$ denote two real separable Hilbert spaces, with their vector norms and their inner products, respectively. We denote by $\mathscr{L}(\mathbb{K} ; \mathbb{Z})$ the set of all linear bounded operators from $\mathbb{K}$ into $\mathbb{t}$, which is equipped with the usual operator norm $\|\cdot\|$. In this paper, we use the symbol $\|\cdot\|$ to denote norms of operators regardless of the spaces potentially involved when no confusion possibly arises. Let $\left(\Omega, \mathscr{F},\left\{\mathscr{F}_{t}\right\}_{t \geq 0}, \mathbf{P}\right)$ be a complete filtered probability space satisfying the usual condition (i.e., it is right continuous and $\mathscr{F}_{0}$ contains all $\mathbf{P}$-null sets). Let $W=$ $(W(t))_{t \geq 0}$ be a $Q$-Wiener process defined on the probability space $\left(\Omega, \mathscr{F},\left\{\mathscr{F}_{t}\right\}_{t \geq 0}, \mathbf{P}\right)$ with the covariance operator $Q$ such that $\operatorname{Tr}(Q)<\infty$. We assume that there exists a complete orthonormal system $\left\{e_{k}\right\}_{k \geq 1}$ in $\mathbb{K}$, a bounded sequence of nonnegative real numbers $\lambda_{k}$ such that $Q e_{k}=\lambda_{k} e_{k}, k=$ $1,2, \ldots$, and a sequence of independent Brownian motions $\left\{\beta_{k}\right\}_{k \geq 1}$ such that $\langle W(t), e\rangle_{\mathbb{K}}=\sum_{k=1}^{\infty} \sqrt{\lambda_{k}}\left\langle e_{k}, e\right\rangle_{\mathbb{K}} \beta_{k}(t), e \in$ $\mathbb{K}, t \geq 0$.

Let $\mathscr{L}_{2}^{0}=\mathscr{L}_{2}\left(Q^{1 / 2} \mathbb{K} ; \mathbb{\square}\right)$ be the space of all HilbertSchmidt operators from $Q^{1 / 2} \mathbb{K}$ to $\mathbb{U}$ with the inner product $\langle\Psi, \phi\rangle_{\mathscr{L}_{2}^{0}}=\operatorname{Tr}\left[\Psi Q \phi^{*}\right]$, where $\phi^{*}$ is the adjoint of the operator $\phi$.

The collection of all strongly measurable, squareintegrable $\mathbb{U}$-valued random variables, denoted by $\mathscr{L}_{2}(\Omega, \mathbb{U})$, is a Banach space equipped with norm $\|x\|_{\mathscr{L}_{2}}=\left(\mathbf{E}\|x\|^{2}\right)^{1 / 2}$. Let $\mathrm{C}\left(J, \mathscr{L}_{2}(\Omega, \mathbb{U})\right)$ be the Banach space of all continuous maps from $J$ to $\mathscr{L}_{2}(\Omega, \mathbb{U})$ satisfying the condition 
$\sup _{t \in J} E\|x(t)\|^{2}<\infty$. An important subspace is given by $\mathscr{L}_{2}^{0}(\Omega, \mathbb{U})=\left\{f \in \mathscr{L}_{2}(\Omega, \mathbb{U}): f\right.$ is $\mathscr{F}_{0}$-measurable $\}$.

Next, to be able to access well-posedness of mild solutions for (3) we need to introduce theory of cosine functions of operators and the second-order abstract Cauchy problem.

Definition 1. (1) The one-parameter family $\{C(t)\}_{t \in \mathbb{R}} \subset \mathscr{L}(\mathbb{U})$ is said to be a strongly continuous cosine family if the following hold:

(i) $C(0)=I, I$ is the identity operators in $\mathbb{H}$;

(ii) $C(t) x$ is continuous in $t$ on $\mathbb{R}$ for any $x \in \mathbb{U}$;

(iii) $C(t+s)+C(t-s)=2 C(t) C(s)$ for all $t, s \in \mathbb{R}$.

(2) The corresponding strongly continuous sine family $\{S(t)\}_{t \in \mathbb{R}} \quad \subset \quad \mathscr{L}(\mathbb{W})$, associated with the given strongly continuous cosine family $\{C(t)\}_{t \in \mathbb{R}} \subset \mathscr{L}(\mathbb{W})$, is defined by

$$
S(t) x=\int_{0}^{t} C(s) x d s, \quad t \in \mathbb{R}, x \in \mathbb{H} .
$$

(3) The infinitesimal generator $A: \mathbb{U} \rightarrow \mathbb{U}$ of $\{C(t)\}_{t \in \mathbb{R}} \mathrm{C}$ $\mathscr{L}(\mathbb{E})$ is given by

$$
A x=\left.\frac{d^{2}}{d t^{2}} C(t) x\right|_{t=0},
$$

for all $x \in D(A)=\left\{x \in \mathbb{H}: C(\cdot) \in C^{2}(\mathbb{R}, \mathbb{H})\right\}$.

It is well known that the infinitesimal generator $A$ is a closed, densely defined operator on $\mathbb{R}$, and the following properties hold; see Travis and Webb [16].

Proposition 2. Suppose that $A$ is the infinitesimal generator of a cosine family of operators $\{C(t)\}_{t \in \mathbb{R}}$. Then, the following hold:

(i) there exist a pair of constants $M_{A} \geq 1$ and $\alpha \geq 0$ such that $\|C(t)\| \leq M_{A} e^{\alpha|t|}$ and hence, $\|S(t)\| \leq M_{A} e^{\alpha|t|}$;

(ii) $A \int_{s}^{r} S(u) x d u=[C(r)-C(s)] x$, for all $0 \leq s \leq r<\infty$;

(iii) there exist $N \geq 1$ such that $\|S(s)-S(r)\| \leq$ $N\left|\int_{s}^{r} e^{\alpha|s|} d s\right|, 0 \leq s \leq r<\infty$.

Thanks to Proposition 2 and the uniform boundedness principle, as a direct consequence we see that both $\{C(t)\}_{t \in J}$ and $\{S(t)\}_{t \in J}$ are uniformly bounded by $\widetilde{M}=M_{A} e^{\alpha|T|}$.

The existence of solutions for the second-order linear abstract Cauchy problem

$$
\begin{gathered}
x^{\prime \prime}(t)=A x(t)+h(t), \quad t \in J, \\
x(0)=z, \quad x^{\prime}(0)=w,
\end{gathered}
$$

where $h: J \rightarrow \mathbb{U}$ is an integrable function has been discussed in [17]. Similarly, the existence of solutions of the semilinear second-order abstract Cauchy problem has been treated in [16].

Definition 3. The function $x(\cdot)$ given by

$$
x(t)=C(t) z+S(t) w+\int_{0}^{t} S(t-s) h(s) d s, \quad t \in J,
$$

is called a mild solution of (6), and that when $z \in \mathbb{A}, x(\cdot)$ is continuously differentiable and

$$
x^{\prime}(t)=A S(t) z+C(t) w+\int_{0}^{t} C(t-s) h(s) d s, \quad t \in J .
$$

For additional details about cosine function theory, we refer the reader to $[16,17]$.

Now we define the abstract phase space $\mathscr{B}$. Assume that $l$ : $J_{0} \rightarrow(0,+\infty)$ is a continuous function with $l_{0}=\int_{J_{0}} l(t) d t<$ $\infty$. For any $a>0$, we define

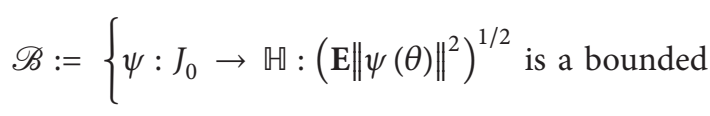

and measurable function on $[-a, 0]$ and

$$
\left.\int_{J_{0}} l(s) \sup _{\theta \in[s, 0]}\left(\mathbf{E}\|\psi(\theta)\|^{2}\right)^{1 / 2} d s<+\infty\right\} .
$$

If $\mathscr{B}$ is endowed with the norm

$$
\|\psi\|_{\mathscr{B}}=\int_{J_{0}} l(s) \sup _{\theta \in[s, 0]}\left(\mathrm{E}\|\psi(\theta)\|^{2}\right)^{1 / 2} d s, \quad \forall \psi \in \mathscr{B},
$$

then it is clear that $\left(\mathscr{B},\|\cdot\|_{\mathscr{B}}\right)$ is a Banach space [18].

Let $J_{T}=(-\infty, T]$. We consider the space

$$
\begin{aligned}
\mathscr{B}_{T}:=\{ & x: J_{T} \longrightarrow \mathbb{U} \text { such that } x_{k} \in \mathrm{C}\left(J_{k}, \mathbb{M}\right) \text { and there } \\
& \text { exist } x\left(t_{k}^{-}\right) \text {and } x\left(t_{k}^{+}\right) \text {with } x\left(t_{k}^{-}\right)=x\left(t_{k}^{+}\right), \\
& \left.x(0)-q\left(x_{t_{1}}, x_{t_{2}}, \ldots, x_{t_{n}}\right)=\varphi \in \mathscr{B}, k=\overline{1, m}\right\},
\end{aligned}
$$

where $x_{k}$ is the restriction of $x$ to $J_{k}=\left(t_{k}, t_{k+1}\right], k=\overline{1, m}$. Set $\|\cdot\|_{T}$ as a seminorm in $\mathscr{B}_{T}$ defined by

$$
\|x\|_{T}=\|\varphi\|_{\mathscr{B}}+\sup _{s \in J}\left(\mathrm{E}\|x(s)\|^{2}\right)^{1 / 2}, \quad x \in \mathscr{B}_{T} .
$$

Lemma 4 (see [2]). Assume that $x \in \mathscr{B}_{T}$, then for $t \in J$, $x_{t} \in \mathscr{B}$. Moreover,

$$
l_{0}\left(\mathbf{E}\|x(t)\|^{2}\right)^{1 / 2} \leq\left\|x_{t}\right\|_{\mathscr{B}} \leq\left\|x_{0}\right\|_{\mathscr{B}}+l_{0} \sup _{s \in[0, t]}\left(\mathbf{E}\|x(s)\|^{2}\right)^{1 / 2} .
$$

Next, we present the Krasnoselskii-Schaefer-type fixed point theorem appearing in [19], which is our main tool.

Lemma 5 (see [19]). Let $\Pi_{1}$ and $\Pi_{2}$ be two operators of $\square$ such that

(a) $\Pi_{1}$ is a contraction, and

(b) $\Pi_{2}$ is completely continuous.

Then, either

(i) the operator equation $\Pi_{1} x+\Pi_{2} x=x$ has a solution, or 
(ii) the set $\Lambda=\left\{x \in \mathbb{H}: \lambda \Pi_{1}(x / \lambda)+\lambda \Pi_{2} x=x\right\}$ is unbounded for $\lambda \in(0,1)$.

Now, we give the definition of mild solution for (3).

Definition 6. An $\mathscr{F}_{t}$-adapted stochastic process $x: J_{T} \rightarrow$ $\mathbb{U}$ is called a mild solution of (3) on $J_{T}$ if $x(0)-$ $q\left(x_{t_{1}}, x_{t_{2}}, \ldots, x_{t_{n}}\right)=x_{0}=\varphi \in \mathscr{B}$ satisfying $q, x_{0} \in$ $\mathscr{L}_{2}^{0}(\Omega, \mathbb{H})$ and $x^{\prime}(0)=x_{1} \in \mathbb{W}$ satisfying $x_{1} \in \mathscr{L}_{2}^{0}(\Omega, \mathbb{H})$; the functions $C(t-s) g\left(s, x_{s}, \int_{0}^{s} \sigma_{1}\left(s, \tau, x_{\tau}\right) d \tau\right)$ and $S(t-$ s) $f\left(s, x_{s}, \int_{0}^{s} \sigma_{2}\left(s, \tau, x_{\tau}\right) d \tau\right)$ are integrable on $J$ such that the following conditions hold:

(i) $\left\{x_{t}: t \in J\right\}$ is a $\mathscr{B}$-valued stochastic process;

(ii) for arbitrary $t \in J, x(t)$ satisfies the following integral equation:

$$
\begin{aligned}
x(t)= & C(t)\left[\varphi(0)+q\left(x_{t_{1}}, x_{t_{2}}, \ldots, x_{t_{n}}\right)(0)\right] \\
& +S(t)\left[x_{1}-g\left(0, x_{0}, 0\right)\right] \\
& +\int_{0}^{t} C(t-s) g\left(s, x_{s}, \int_{0}^{s} \sigma_{1}\left(s, \tau, x_{\tau}\right) d \tau\right) d s \\
& +\int_{0}^{t} S(t-s) f\left(s, x_{s}, \int_{0}^{s} \sigma_{2}\left(s, \tau, x_{\tau}\right) d \tau\right) d s \\
& +\sum_{0<t_{k}<t} C\left(t-t_{k}\right) I_{k}^{1}\left(x\left(t_{k}^{-}\right)\right) \\
& +\int_{0}^{t} S(t-s) \int_{-\infty}^{s} \sigma\left(s, \tau, x_{\tau}\right) d W(\tau) d s \\
& +\sum_{0<t_{k}<t} S\left(t-t_{k}\right) I_{k}^{2}\left(x\left(t_{k}^{-}\right)\right) ;
\end{aligned}
$$

(iii) $\Delta x\left(t_{k}\right)=I_{k}^{1}\left(x\left(t_{k}^{-}\right)\right), \Delta x^{\prime}\left(t_{k}\right)=I_{k}^{2}\left(x\left(t_{k}^{-}\right)\right), k=\overline{1, m}$. tions.

In this paper, we will work under the following assump-

(H1) The cosine family of operators $\{C(t)\}_{t \in J}$ on $\mathbb{H}$ and the corresponding sine family $\{S(t)\}_{t \in J}$ are compact for $t>0$, and there exist positive constants $M_{C}, M_{S}$ such that for all $t \in J$,

$$
\|C(t)\|^{2} \leq M_{C}, \quad\|S(t)\|^{2} \leq M_{S} .
$$

(H2) There exists a positive constant $M_{\sigma_{1}}$ such that for all $t, s \in J, x, y \in \mathscr{B}$

$$
\mathbf{E}\left\|\int_{0}^{t}\left[\sigma_{1}(t, s, x)-\sigma_{1}(t, s, y)\right] d s\right\|^{2} \leq M_{\sigma_{1}}\|x-y\|_{\mathscr{B}}^{2} .
$$

(H3) The function $g: J \times \mathscr{B} \times \mathbb{H} \rightarrow \mathbb{H}$ is continuous and there exists a positive constant $M_{g}$ such that for all $t \in J, x_{1}, x_{2} \in \mathscr{B}, y_{1}, y_{2} \in \mathbb{H}$

$$
\begin{aligned}
\mathbf{E} \| g( & \left.t, x_{1}, y_{1}\right)-g\left(t, x_{2}, y_{2}\right) \|^{2} \\
& \leq M_{g}\left(\left\|x_{1}-\left.x_{2}\right|_{\mathscr{B}} ^{2}+\mathbf{E}\right\| y_{1}-y_{2} \|^{2}\right) .
\end{aligned}
$$

(H4) For each $(t, s) \in J \times J$, the function $\sigma_{2}(t, s, \cdot): \mathscr{B} \rightarrow$ $\mathbb{H}$ is continuous and for each $x \in \mathscr{B}$, the function $\sigma_{2}(\cdot, \cdot, x): J \times J \rightarrow \mathbb{H}$ is strongly measurable. There exists an integrable function $\mu: J \rightarrow[0, \infty)$ and a positive constant $M_{\sigma}$ such that

$$
\mathbf{E}\left\|\sigma_{2}(t, s, x)\right\|^{2} \leq M_{\sigma} \mu(s) \Omega_{1}\left(\|x\|_{\mathscr{B}}^{2}\right)
$$

where $\Omega_{1}:[0, \infty) \rightarrow(0, \infty)$ is a continuous nondecreasing function. Assume that the finite bound of $\int_{0}^{t} M_{\sigma} \mu(s) d s$ is $M_{\mu}$.

(H5) The function $f: J \times \mathscr{B} \times \mathbb{M} \rightarrow \mathbb{M}$ satisfies the following Carathéodory conditions:

(i) $t \rightarrow f(t, x, y)$ is measurable for each $(x, y) \in$ $\mathscr{B} \times \mathbb{H}$

(ii) $(x, y) \rightarrow f(t, x, y)$ is continuous for almost all $t \in J$

(iii) $\mathbf{E}\|f(t, x, y)\|^{2} \leq \eta(t) \Omega_{2}\left(\|x\|_{\mathscr{B}}^{2}+\mathbf{E}\|y\|^{2}\right)$ for almost all $t \in J, y \in \mathbb{H}$, where $\eta \in \mathscr{L}^{1}(J,[0, \infty))$ and $\Omega_{2}:[0, \infty) \rightarrow(0, \infty)$ is a continuous increasing function.

(H6) The functions $I_{k}^{1}, I_{k}^{2} \in \mathrm{C}(\mathbb{H}, \mathbb{H})$ and there exist positive constants $M_{I_{k}^{1}}, M_{I_{k}^{2}}$ such that for all $x \in \mathbb{H}$,

$$
\mathbf{E}\left\|I_{k}^{1}(x)\right\|^{2} \leq M_{I_{k}^{1}}, \quad \mathbf{E}\left\|I_{k}^{2}(x)\right\|^{2} \leq M_{I_{k}^{2}}, \quad k=\overline{1, m} .
$$

(H7) For each $\varphi \in \mathscr{B}, h(t)=\lim _{c \rightarrow \infty} \int_{-c}^{0} \sigma(t, s, \varphi) d W(s)$ exists and is continuous. Further, there exists a positive constant $M_{h}$ such that

$$
\mathbf{E}\|h(t)\|^{2} \leq M_{h}
$$

(H8) The function $\sigma: J \times J \times \mathscr{B} \rightarrow \mathscr{L}(\mathbb{K}, \mathbb{H})$ is continuous and there exists a positive constant $M_{\sigma}$ such that for all $s, t \in J$ and $\nu \in \mathscr{B}$

$$
\mathbf{E}\|\sigma(t, s, v)\|_{\mathscr{L}_{2}^{0}}^{2} \leq M_{\sigma}
$$

(H9) The function $q: \mathscr{B}^{n} \rightarrow \mathscr{B}$ is continuous and there exists a positive constant $M_{q}$ such that for all $x, y \in$ $\mathscr{B}, t \in J_{0}$

$$
\mathbf{E}\left\|q\left(x_{t_{1}}, x_{t_{2}}, \ldots, x_{t_{n}}\right)(t)\right\|^{2} \leq M_{q} .
$$

(H10) Assume that the following relationship holds:

$$
\begin{aligned}
& \int_{0}^{T} \widehat{\Omega}(s) d s \leq \int_{M_{1}}^{\infty} \frac{d s}{\Omega_{1}(s)+\Omega_{2}(s)}, \\
& \widehat{\Omega}(t):=\max \left\{M_{2} \eta(t), M_{\sigma} T \mu(t)\right\},
\end{aligned}
$$




$$
\begin{aligned}
C_{1}:=T \sup _{(t, s) \in J \times J} \sigma_{1}^{2}(t, s, 0), \quad C_{2}:=\sup _{t \in J}\|g(t, 0,0)\|^{2}, \\
28 l_{0}^{2} T^{2} M_{C} M_{g}\left(1+2 M_{\sigma_{1}}\right)<1, \\
M_{1}:=\frac{2\|\varphi\|_{\mathscr{B}}^{2}+2 l_{0}^{2} M^{\star}}{1-28 l_{0}^{2} T^{2} M_{C} M_{g}\left(1+2 M_{\sigma_{1}}\right)}, \\
M_{2}:=\frac{14 l_{0}^{2} T M_{S}}{1-28 l_{0}^{2} T^{2} M_{C} M_{g}\left(1+2 M_{\sigma_{1}}\right)} \\
M^{\star}:=14 M_{C}\left[\mathbf{E}\|\varphi(0)\|^{2}+M_{q}\right] \\
+14 M_{S}\left[\mathbf{E}\left\|x_{1}\right\|^{2}+2\left(M_{g}\|\varphi\|_{\mathscr{B}}^{2}+C_{2}\right)\right] \\
+28 T^{2} M_{C} M_{g} C_{1}+14 T^{2} M_{C} C_{2} \\
+14 T^{2} M_{S}\left(M_{h}+T T_{r}(Q) M_{\sigma}\right) \\
+7 M_{C} \sum_{k=1}^{m} M_{I_{k}^{1}}+7 M_{S} \sum_{k=1}^{m} M_{I_{k}^{2}}
\end{aligned}
$$

\section{Main Results}

In this section, we will investigate the existence of mild solutions for a class of second- order impulsive neutral stochastic integrodifferential equations with nonlocal conditions and infinite delays in Hilbert spaces.

We consider the operator $\Pi: \mathscr{B}_{T} \rightarrow \mathscr{B}_{T}$ defined by

$$
\Pi x(t)=\varphi(t)+q\left(x_{t_{1}}, x_{t_{2}}, \ldots, x_{t_{n}}\right)(t), \quad t \in J_{0} ;
$$

$$
\begin{aligned}
\Pi x(t)= & C(t)\left[\varphi(0)+q\left(x_{t_{1}}, x_{t_{2}}, \ldots, x_{t_{n}}\right)(0)\right] \\
& +S(t)\left[x_{1}-g\left(0, x_{0}, 0\right)\right] \\
& +\int_{0}^{t} C(t-s) g\left(s, x_{s}, \int_{0}^{s} \sigma_{1}\left(s, \tau, x_{\tau}\right) d \tau\right) d s \\
& +\int_{0}^{t} S(t-s) f\left(s, x_{s}, \int_{0}^{s} \sigma_{2}\left(s, \tau, x_{\tau}\right) d \tau\right) d s \\
& +\sum_{0<t_{k}<t} C\left(t-t_{k}\right) I_{k}^{1}\left(x\left(t_{k}^{-}\right)\right) \\
& +\int_{0}^{t} S(t-s)\left[h(s)+\int_{0}^{s} \sigma\left(s, \tau, x_{\tau}\right) d W(\tau)\right] d s \\
& +\sum_{0<t_{k}<t} S\left(t-t_{k}\right) I_{k}^{2}\left(x\left(t_{k}^{-}\right)\right), \quad t \in J .
\end{aligned}
$$

For $\varphi \in \mathscr{B}$, we defined $\widetilde{\varphi}$ by

$$
\tilde{\varphi}(t)= \begin{cases}\varphi(t) & \text { if } t \in J_{0}, \\ C(t)\left[\varphi(0)+q\left(x_{t_{1}}, x_{t_{2}}, \ldots, x_{t_{n}}\right)(0)\right] & \text { if } t \in J .\end{cases}
$$

Then $\tilde{\varphi} \in \mathscr{B}_{T}$.
Let $x(t)=u(t)+\widetilde{\varphi}(t), t \in J_{T}$. It is easy to see that $x$ satisfies (14) if and only if $u$ satisfies $u_{0}=0, x^{\prime}(0)=x_{1}=u^{\prime}(0)=u_{1}$, and

$$
\begin{aligned}
u(t)= & S(t)\left[u_{1}-g\left(0, \widetilde{\varphi}_{0}, 0\right)\right] \\
& +\int_{0}^{t} C(t-s) g\left(s, u_{s}+\widetilde{\varphi}_{s}, \int_{0}^{s} \sigma_{1}\left(s, \tau, u_{\tau}+\widetilde{\varphi}_{\tau}\right) d \tau\right) d s \\
& +\int_{0}^{t} S(t-s) f\left(s, u_{s}+\widetilde{\varphi}_{s}, \int_{0}^{s} \sigma_{2}\left(s, \tau, u_{\tau}+\widetilde{\varphi}_{\tau}\right) d \tau\right) d s \\
& +\int_{0}^{t} S(t-s) \int_{-\infty}^{s} \sigma\left(s, \tau, u_{\tau}+\widetilde{\varphi}_{\tau}\right) d W(\tau) d s \\
& +\sum_{0<t_{k}<t} C\left(t-t_{k}\right) I_{k}^{1}\left(u\left(t_{k}^{-}\right)+\widetilde{\varphi}\left(t_{k}^{-}\right)\right) \\
& +\sum_{0<t_{k}<t} S\left(t-t_{k}\right) I_{k}^{2}\left(u\left(t_{k}^{-}\right)+\widetilde{\varphi}\left(t_{k}^{-}\right)\right), \quad t \in J .
\end{aligned}
$$
have

Let $\mathscr{B}_{T}^{0}=\left\{u \in \mathscr{B}_{T}: u_{0}=0 \in \mathscr{B}\right\}$. For any $u \in \mathscr{B}_{T}^{0}$, we

$$
\|u\|_{T}=\left\|u_{0}\right\|_{\mathscr{B}}+\sup _{s \in J}\left(\mathbf{E}\|u(s)\|^{2}\right)^{1 / 2}=\sup _{s \in J}\left(\mathbf{E}\|u(s)\|^{2}\right)^{1 / 2},
$$

and thus $\left(\mathscr{B}_{T}^{0},\|\cdot\|_{T}\right)$ is a Banach space. Set

$$
B_{r}=\left\{u \in \mathscr{B}_{T}^{0}:\|u\|_{T}^{2} \leq r\right\} \quad \text { for some } r \geq 0 \text {. }
$$

Then $B_{r} \subseteq \mathscr{B}_{T}^{0}$ is uniformly bounded, and for $u \in B_{r}$, by Lemma 4, we have

$$
\begin{aligned}
\left\|u_{t}+\widetilde{\varphi}_{t}\right\|_{\mathscr{B}}^{2} \leq & 2\left(\left\|u_{t}\right\|_{\mathscr{B}}^{2}+\left\|\widetilde{\varphi}_{t}\right\|_{\mathscr{B}}^{2}\right) \\
\leq & 4\left(l_{0}^{2} \sup _{s \in[0, t]}\left(\mathbf{E}\|u(s)\|^{2}\right)+\left\|u_{0}\right\|_{\mathscr{B}}^{2}\right. \\
& \left.+l_{0}^{2} \sup _{s \in[0, t]}\left(\mathbf{E}\|\widetilde{\varphi}(s)\|^{2}\right)+\left\|\widetilde{\varphi}_{0}\right\|_{\mathscr{B}}^{2}\right) \\
\leq & 4 l_{0}^{2}\left(r+2 M_{C}\left[\mathbf{E}\|\varphi(0)\|^{2}+M_{q}\right]\right)+4\|\widetilde{\varphi}\|_{\mathscr{B}}^{2}:=r^{\star} .
\end{aligned}
$$

Define the map $\bar{\Pi}: \mathscr{B}_{T}^{0} \rightarrow \mathscr{B}_{T}^{0}$ defined by $\bar{\Pi} u(t)=0$, for $t \in J_{0}$ and

$\bar{\Pi} u(t)$

$$
\begin{aligned}
= & S(t)\left[u_{1}-g\left(0, \tilde{\varphi}_{0}, 0\right)\right] \\
& +\int_{0}^{t} C(t-s) g\left(s, u_{s}+\widetilde{\varphi}_{s}, \int_{0}^{s} \sigma_{1}\left(s, \tau, u_{\tau}+\widetilde{\varphi}_{\tau}\right) d \tau\right) d s
\end{aligned}
$$




$$
\begin{aligned}
& +\int_{0}^{t} S(t-s) f\left(s, u_{s}+\widetilde{\varphi}_{s}, \int_{0}^{s} \sigma_{2}\left(s, \tau, u_{\tau}+\widetilde{\varphi}_{\tau}\right) d \tau\right) d s \\
& +\int_{0}^{t} S(t-s)\left[h(s)+\int_{0}^{s} \sigma\left(s, \tau, u_{\tau}+\widetilde{\varphi}_{\tau}\right) d W(\tau)\right] d s \\
& +\sum_{0<t_{k}<t} C\left(t-t_{k}\right) I_{k}^{1}\left(u\left(t_{k}^{-}\right)+\widetilde{\varphi}\left(t_{k}^{-}\right)\right) \\
& +\sum_{0<t_{k}<t} S\left(t-t_{k}\right) I_{k}^{2}\left(u\left(t_{k}^{-}\right)+\widetilde{\varphi}\left(t_{k}^{-}\right)\right), \quad t \in J .
\end{aligned}
$$

Now, we decompose $\bar{\Pi}$ as $\bar{\Pi}=\bar{\Pi}_{1}+\bar{\Pi}_{2}$, where

$$
\begin{aligned}
& \bar{\Pi}_{1} u(t) \\
& =S(t)\left[u_{1}-g\left(0, \tilde{\varphi}_{0}, 0\right)\right] \\
& \quad+\int_{0}^{t} C(t-s) g\left(s, u_{s}+\tilde{\varphi}_{s}, \int_{0}^{s} \sigma_{1}\left(s, \tau, u_{\tau}+\widetilde{\varphi}_{\tau}\right) d \tau\right) d s, \quad t \in J, \\
& \bar{\Pi}_{2} u(t) \\
& \quad \int_{0}^{t} S(t-s) f\left(s, u_{s}+\tilde{\varphi}_{s}, \int_{0}^{s} \sigma_{2}\left(s, \tau, u_{\tau}+\widetilde{\varphi}_{\tau}\right) d \tau\right) d s \\
& \quad+\int_{0}^{t} S(t-s)\left[h(s)+\int_{0}^{s} \sigma\left(s, \tau, u_{\tau}+\tilde{\varphi}_{\tau}\right) d W(\tau)\right] d s \\
& \quad+\sum_{0<t_{k}<t} C\left(t-t_{k}\right) I_{k}^{1}\left(u\left(t_{k}^{-}\right)+\widetilde{\varphi}\left(t_{k}^{-}\right)\right) \\
& \quad+\sum_{0<t_{k}<t} S\left(t-t_{k}\right) I_{k}^{2}\left(u\left(t_{k}^{-}\right)+\widetilde{\varphi}\left(t_{k}^{-}\right)\right), \quad t \in J .
\end{aligned}
$$

Obviously, the operator $\Pi$ having a fixed point is equivalent to $\bar{\Pi}$ having one. Now, we will show that the operators $\bar{\Pi}_{1}, \bar{\Pi}_{2}$ satisfy all the conditions of Lemma 5.

Lemma 7. Let the assumptions (H1)-(H10) hold. Then $\bar{\Pi}_{1}$ is contractive.

Proof. Let $u, v \in \mathscr{B}_{T}^{0}$. Then, by our assumptions and Lemma 4, for each $t \in J$, we have

$$
\begin{aligned}
& \mathbf{E}\left\|\left(\bar{\Pi}_{1} u\right)(t)-\left(\bar{\Pi}_{1} v\right)(t)\right\|^{2} \\
& \quad \leq T^{2} M_{C} M_{g}\left(1+M_{\sigma_{1}}\right)\left\|u_{t}-v_{t}\right\|_{\mathscr{B}}^{2} \\
& \quad \leq 2 l_{0}^{2} T^{2} M_{C} M_{g}\left(1+M_{\sigma_{1}}\right) \sup _{s \in J} \mathrm{E}\|u(s)-v(s)\|^{2} .
\end{aligned}
$$

Since $\left\|u_{0}\right\|_{\mathscr{B}}^{2}=0$ and $\left\|v_{0}\right\|_{\mathscr{B}}^{2}=0$. Taking the supremum over $t$, we obtain

$$
\left\|\left(\bar{\Pi}_{1} u\right)-\left(\bar{\Pi}_{1} v\right)\right\|_{T}^{2} \leq 2 l_{0}^{2} T^{2} M_{C} M_{g}\left(1+M_{\sigma_{1}}\right)\|u-v\|_{T}^{2} .
$$

By assumption (H10), we conclude that $\bar{\Pi}_{1}$ is a contraction on $\mathscr{B}_{T}^{0}$. Thus we have completed the proof of Lemma 7.

Lemma 8. Let the assumptions (H1)-(H10) hold. Then $\bar{\Pi}_{2}$ is completely continuous.

Proof. The proof of the lemma is long. Therefore it is convenient to divide it into the following four steps.

Step 1. $\bar{\Pi}_{2}$ maps bounded sets to bounded sets in $\mathscr{B}_{\mathrm{T}}^{0}$.

Indeed, it is enough to show that there exists a positive constant $\Delta$ such that for each $u \in B_{r}=\left\{u \in \mathscr{B}_{T}^{0}:\|u\|_{T}^{2} \leq\right.$ $r\}$, one has $\mathbf{E}\left\|\bar{\Pi}_{2} u\right\|_{T}^{2} \leq \Delta$. By our assumptions, Hölder's inequality, and Burkholder-Davis-Gundy's inequality, for $t \epsilon$ $J$, we have

$$
\begin{aligned}
& \mathbf{E}\left\|\bar{\Pi}_{2} u(t)\right\|^{2} \\
& \leq 4 \mathbf{E}\left\|\int_{0}^{t} S(t-s) f\left(s, u_{s}+\widetilde{\varphi}_{s}, \int_{0}^{s} \sigma_{2}\left(s, \tau, u_{\tau}+\widetilde{\varphi}_{\tau}\right) d \tau\right) d s\right\|^{2} \\
& +4 \mathbf{E}\left\|\int_{0}^{t} S(t-s)\left[h(s)+\int_{0}^{s} \sigma\left(s, \tau, u_{\tau}+\widetilde{\varphi}_{\tau}\right) d W(\tau)\right] d s\right\|^{2} \\
& +4 \mathbf{E}\left\|\sum_{0<t_{k}<t} C\left(t-t_{k}\right) I_{k}^{1}\left(u\left(t_{k}^{-}\right)+\widetilde{\varphi}\left(t_{k}^{-}\right)\right)\right\|^{2} \\
& +4 \mathbf{E}\left\|\sum_{0<t_{k}<t} S\left(t-t_{k}\right) I_{k}^{2}\left(u\left(t_{k}^{-}\right)+\widetilde{\varphi}\left(t_{k}^{-}\right)\right)\right\|^{2} \\
& \leq 4\left[M_{S} T \Omega_{2}\left(r^{\star}+M_{\mu^{\prime}} \Omega_{1}\left(r^{\star}\right)\right) \int_{0}^{T} \eta(s) d s\right. \\
& +M_{S} T^{2}\left(2 M_{h}+2 T r(Q) T M_{\sigma}\right) \\
& \left.+M_{C} \sum_{k=1}^{m} M_{I_{k}^{1}}+M_{S} \sum_{k=1}^{m} M_{I_{k}^{2}}\right]:=\Delta .
\end{aligned}
$$

Then, for each $u \in B_{r}=\left\{u \in \mathscr{B}_{T}^{0}:\|u\|_{T}^{2} \leq r\right\}$, we get $\mathbf{E}\left\|\bar{\Pi}_{2} u\right\|_{T}^{2} \leq \Delta$.

Step 2. $\bar{\Pi}_{2}$ maps bounded sets into equicontinuous sets of $\mathscr{B}_{T}^{0}$. Let $0<v_{1}<v_{2} \leq T$, for each $u \in B_{r}=\{u \in$ $\left.\mathscr{B}_{T}^{0}:\|u\|_{T}^{2} \leq r\right\}$. Let $v_{1}, v_{2} \in J \backslash\left\{t_{1}, t_{2}, \ldots, t_{m}\right\}$. Then, by our assumptions, Hölder's inequality, and Burkholder-DavisGundy's inequality, we get

$$
\begin{aligned}
& \mathbf{E}\left\|\left(\bar{\Pi}_{2} u\right)\left(\nu_{1}\right)-\left(\bar{\Pi}_{2} u\right)\left(v_{2}\right)\right\|^{2} \\
& \leq 8 \mathbf{E} \| \int_{0}^{v_{1}}\left[S\left(\nu_{1}-s\right)-S\left(\nu_{2}-s\right)\right] f \\
& \quad \times\left(s, u_{s}+\widetilde{\varphi}_{s}, \int_{0}^{s} \sigma_{2}\left(s, \tau, u_{\tau}+\widetilde{\varphi}_{\tau}\right) d \tau\right) d s \|^{2} \\
& +8 \mathbf{E} \| \int_{v_{1}}^{v_{2}} S\left(\nu_{2}-s\right) f \\
& \quad \times\left(s, u_{s}+\widetilde{\varphi}_{s}, \int_{0}^{s} \sigma_{2}\left(s, \tau, u_{\tau}+\widetilde{\varphi}_{\tau}\right) d \tau\right) d s \|^{2}
\end{aligned}
$$




$$
\begin{aligned}
& +8 \mathbf{E} \| \int_{0}^{\nu_{1}}\left[S\left(\nu_{1}-s\right)-S\left(\nu_{2}-s\right)\right] \\
& \times\left[h(s)+\int_{0}^{s} \sigma\left(s, \tau, u_{\tau}+\widetilde{\varphi}_{\tau}\right) d W(\tau)\right] d s \|^{2} \\
& +8 \mathbf{E} \| \int_{v_{1}}^{v_{2}} S\left(\nu_{2}-s\right) \\
& \times\left[h(s)+\int_{0}^{s} \sigma\left(s, \tau, u_{\tau}+\widetilde{\varphi}_{\tau}\right) d W(\tau)\right] d s \|^{2} \\
& +8 \mathbf{E} \| \sum_{0<t_{k}<\nu_{1}}\left[C\left(v_{1}-t_{k}\right)-C\left(\nu_{2}-t_{k}\right)\right] \\
& \times I_{k}^{1}\left(u\left(t_{k}^{-}\right)+\widetilde{\varphi}\left(t_{k}^{-}\right)\right) \|^{2} \\
& +8 \mathbf{E}\left\|\sum_{\nu_{1}<t_{k}<v_{2}} C\left(\nu_{2}-t_{k}\right) I_{k}^{1}\left(u\left(t_{k}^{-}\right)+\widetilde{\varphi}\left(t_{k}^{-}\right)\right)\right\|^{2} \\
& +8 \mathrm{E} \| \sum_{0<t_{k}<v_{1}}\left[S\left(\nu_{1}-t_{k}\right)-S\left(v_{2}-t_{k}\right)\right] \\
& \times I_{k}^{2}\left(u\left(t_{k}^{-}\right)+\widetilde{\varphi}\left(t_{k}^{-}\right)\right) \|^{2} \\
& +8 \mathbf{E}\left\|\sum_{\nu_{1}<t_{k}<v_{2}} S\left(\nu_{2}-t_{k}\right) I_{k}^{2}\left(u\left(t_{k}^{-}\right)+\widetilde{\varphi}\left(t_{k}^{-}\right)\right)\right\|^{2} \\
& \leq 8 T \int_{0}^{\nu_{1}}\left\|S\left(\nu_{1}-s\right)-S\left(\nu_{2}-s\right)\right\|^{2} \eta(s) \Omega_{2}\left(r^{\star}\right) d s \\
& +8 T\left[M_{h}+\operatorname{Tr}(Q) T M_{\sigma}\right] \\
& \times \int_{0}^{\nu_{1}}\left\|S\left(\nu_{1}-s\right)-S\left(\nu_{2}-s\right)\right\|^{2} d s \\
& +8\left(\nu_{2}-v_{1}\right) \int_{v_{1}}^{v_{2}}\left\|S\left(\nu_{2}-s\right)\right\|^{2} \eta(s) \Omega_{2}\left(r^{\star}\right) d s \\
& +8\left(v_{2}-v_{1}\right)\left[M_{h}+\operatorname{Tr}(Q)\left(v_{2}-v_{1}\right) M_{\sigma}\right] \\
& \times \int_{\nu_{1}}^{\nu_{2}}\left\|S\left(\nu_{2}-s\right)\right\|^{2} d s \\
& +8 \sum_{0<t_{k}<\nu_{1}}\left\|C\left(v_{1}-t_{k}\right)-C\left(v_{2}-t_{k}\right)\right\|^{2} M_{I_{k}^{1}} \\
& +8 \sum_{v_{1}<t_{k}<v_{2}}\left\|C\left(\nu_{2}-t_{k}\right)\right\|^{2} M_{I_{k}^{1}} \\
& +8 \sum_{0<t_{k}<\nu_{1}}\left\|S\left(\nu_{1}-t_{k}\right)-S\left(\nu_{2}-t_{k}\right)\right\|^{2} M_{I_{k}^{2}} \\
& +8 \sum_{v_{1}<t_{k}<v_{2}}\left\|S\left(\nu_{2}-t_{k}\right)\right\|^{2} M_{I_{k}^{2}}
\end{aligned}
$$

The right-hand side of the above inequality which is independent of $u \in B_{r}$ tends to zero as $\nu_{2}-v_{1} \rightarrow 0$. Thus, the set $\left\{\bar{\Pi}_{2}(u): u \in B_{r}\right\}$ is equicontinuous. (Note that we consider only the case $0<v_{1}<v_{2} \leq T$; this proves the equicontinuity for the case where $t \neq t_{k}, k=\overline{1, m}$. Easily we prove the equicontinuity for the case where $t=t_{k}$. And also the other cases $v_{1}<v_{2} \leq 0$ and $v_{1} \leq 0 \leq v_{2} \leq T$ are very simple.)

Step 3. $\bar{\Pi}_{2}: \mathscr{B}_{T}^{0} \rightarrow \mathscr{B}_{T}^{0}$ is continuous.

Let $\left\{u^{(n)}\right\}_{n=0}^{\infty} \subseteq \mathscr{B}_{T}^{0}$, with $u^{(n)} \rightarrow u$ in $\mathscr{B}_{T}^{0}$. Then, there is a number $r>0$ such that $\left\|u^{(n)}\right\| \leq r$ for all $n$ and a.e. $t \in J$, so $u^{(n)} \in B_{r}$ and $u \in B_{r}$. In view of (29), for $t \in J$, we have $\left\|u_{t}+\widetilde{\varphi}_{t}\right\|_{\mathscr{B}}^{2} \leq r^{\star}$.

By Definition 6, the assumptions (H5)-(H8),

$$
\begin{aligned}
f\left(t, u_{t}^{(n)}+\widetilde{\varphi}_{t}, \int_{0}^{t} \sigma_{2}\left(s, \tau, u_{\tau}^{(n)}+\widetilde{\varphi}_{\tau}\right) d \tau\right) \\
\quad \longrightarrow f\left(t, u_{t}+\widetilde{\varphi}_{t}, \int_{0}^{t} \sigma_{2}\left(s, \tau, u_{\tau}+\widetilde{\varphi}_{\tau}\right) d \tau\right)
\end{aligned}
$$

for each $t \in J$, and since

$$
\begin{gathered}
\mathbf{E} \| f\left(t, u_{t}^{(n)}+\widetilde{\varphi}_{t}, \int_{0}^{t} \sigma_{2}\left(s, \tau, u_{\tau}^{(n)}+\widetilde{\varphi}_{\tau}\right) d \tau\right) \\
-f\left(t, u_{t}+\widetilde{\varphi}_{t}, \int_{0}^{t} \sigma_{2}\left(s, \tau, u_{\tau}+\widetilde{\varphi}_{\tau}\right) d \tau\right) \|^{2} \\
\leq 2 \eta(t) \Omega_{2}\left(r^{\star}\right)
\end{gathered}
$$

thanks to the dominated convergence theorem, we obtain that

$$
\begin{gathered}
\left\|\bar{\Pi}_{2} u^{(n)}-\bar{\Pi}_{2} u\right\|_{T}^{2} \\
\leq 4 T M_{S} \int_{0}^{t} \mathbf{E} \| f\left(t, u_{t}^{(n)}+\widetilde{\varphi}_{t}, \int_{0}^{t} \sigma_{2}\left(s, \tau, u_{\tau}^{(n)}+\widetilde{\varphi}_{\tau}\right) d \tau\right) \\
\quad-f\left(t, u_{t}+\widetilde{\varphi}_{t}, \int_{0}^{t} \sigma_{2}\left(s, \tau, u_{\tau}+\widetilde{\varphi}_{\tau}\right) d \tau\right) \|^{2} d s \\
+4 T M_{S} \operatorname{Tr}(Q) \int_{0}^{t} \int_{0}^{s} \mathbf{E} \| \sigma\left(s, \tau, u_{\tau}^{(n)}+\widetilde{\varphi}_{\tau}\right) \\
+4 \sum_{0<t_{k}<t}\left\|C\left(t-t_{k}\right)\right\|^{2} \mathbf{E} \| I_{k}^{1}\left(u^{(n)}\left(t_{k}^{-}\right)+\widetilde{\varphi}\left(t_{k}^{-}\right)\right) \\
\quad-I_{k}^{1}\left(u\left(t_{k}^{-}\right)+\widetilde{\varphi}\left(t_{k}^{-}\right)\right) \|^{2} \\
+4 \sum_{0<t_{k}<t}\left\|S\left(t-t_{k}\right)\right\|^{2} \mathbf{E} \| I_{k}^{2}\left(u^{(n)}\left(t_{k}^{-}\right)+\widetilde{\varphi}\left(t_{k}^{-}\right)\right) \\
\quad-I_{k}^{2}\left(u\left(t_{k}^{-}\right)+\widetilde{\varphi}\left(t_{k}^{-}\right)\right) \|^{2} \stackrel{n \rightarrow \infty}{\longrightarrow} 0 .
\end{gathered}
$$

Thus, $\bar{\Pi}_{2}$ is continuous.

Step 4. $\bar{\Pi}_{2}$ maps $B_{r}$ onto a precompact set in $B_{r}$. That is, for every fixed $t \in J$, the set $\bar{V}(t)=\left\{\left(\bar{\Pi}_{2} u\right)(t): u \in B_{r}\right\}$ is precompact in $B_{r}$. 
It is obvious that $\bar{V}(0)=\left\{\left(\bar{\Pi}_{2} u\right)(0)\right\}$ is precompact. Let $0<t \leq T$ be fixed and $\epsilon$ a real number satisfying $\epsilon \in(0, t)$. For $u \in B_{r}$, we define the operator

$$
\begin{aligned}
& \left(\bar{\Pi}_{2}^{\epsilon} u\right)(t) \\
& =\int_{0}^{t-\epsilon} S(t-s) f\left(s, u_{s}+\widetilde{\varphi}_{s}, \int_{0}^{s} \sigma_{2}\left(s, \tau, u_{\tau}+\widetilde{\varphi}_{\tau}\right) d \tau\right) d s \\
& \quad+\int_{0}^{t-\epsilon} S(t-s)\left[h(s)+\int_{0}^{s} \sigma\left(s, \tau, u_{\tau}+\widetilde{\varphi}_{\tau}\right) d W(\tau)\right] d s \\
& \quad+\sum_{0<t_{k}<t-\epsilon} C\left(t-t_{k}\right) I_{k}^{1}\left(u\left(t_{k}^{-}\right)+\widetilde{\varphi}\left(t_{k}^{-}\right)\right) \\
& \quad+\sum_{0<t_{k}<t-\epsilon} S\left(t-t_{k}\right) I_{k}^{2}\left(u\left(t_{k}^{-}\right)+\widetilde{\varphi}\left(t_{k}^{-}\right)\right) .
\end{aligned}
$$

Since $C(t), S(t), t>0$, are compact, it follows that the set $\bar{V}_{\epsilon}(t)=\left\{\left(\bar{\Pi}_{2}^{\epsilon} u\right)(t): u \in B_{r}\right\}$ is precompact in $\mathbb{H}$, for every $\epsilon, \epsilon \in(0, t)$. Moreover, also by Hölder's inequality and Burkholder-Davis-Gundy's inequality, for each $u \in B_{r}$, we get

$$
\begin{aligned}
& \mathbf{E}\left\|\left(\bar{\Pi}_{2} u\right)(t)-\left(\bar{\Pi}_{2}^{\epsilon} u\right)(t)\right\|^{2} \\
& \leq 4 \epsilon \int_{t-\epsilon}^{t}\|S(t-s)\|^{2} \\
& \times \mathbf{E}\left\|f\left(s, u_{s}+\widetilde{\varphi}_{s}, \int_{0}^{s} \sigma_{2}\left(s, \tau, u_{\tau}+\widetilde{\varphi}_{\tau}\right) d \tau\right)\right\|^{2} d s \\
& +4 \epsilon \int_{t-\epsilon}^{t}\|S(t-s)\|^{2} \\
& \times \mathbf{E}\left\|\left[h(s)+\int_{0}^{s} \sigma\left(s, \tau, u_{\tau}+\widetilde{\varphi}_{\tau}\right) d W(\tau)\right]\right\|^{2} d s \\
& +4 \sum_{t-\epsilon<t_{k}<t}\left\|C\left(t-t_{k}\right)\right\|^{2} \mathbf{E}\left\|I_{k}^{1}\left(u\left(t_{k}^{-}\right)+\widetilde{\varphi}\left(t_{k}^{-}\right)\right)\right\|^{2} \\
& +4 \sum_{t-\epsilon<t_{k}<t}\left\|S\left(t-t_{k}\right)\right\|^{2} \mathbf{E}\left\|I_{k}^{2}\left(u\left(t_{k}^{-}\right)+\widetilde{\varphi}\left(t_{k}^{-}\right)\right)\right\|^{2} \\
& \leq 4 \epsilon M_{S}\left(\int_{t-\epsilon}^{t} \eta(s) \Omega_{2}\left(r^{\star}\right) d s+2 M_{h} T+2 T^{2} \operatorname{Tr}(Q) M_{\sigma}\right) \\
& +4 M_{C} \sum_{t-\epsilon<t_{k}<t} M_{I_{k}^{1}}+4 M_{S} \sum_{t-\epsilon<t_{k}<t} M_{I_{k}^{2}} \stackrel{\epsilon \rightarrow 0}{\longrightarrow} 0,
\end{aligned}
$$

and there are precompact sets arbitrarily close to the set $\left\{\left(\bar{\Pi}_{2} u\right)(t): u \in B_{r}\right\}$. Thus, the set $\left\{\left(\bar{\Pi}_{2} u\right)(t): u \in B_{r}\right\}$ is precompact in $B_{r}$.

Finally, by the Arzelá-Ascoli theorem, we can conclude that the operator $\bar{\Pi}_{2}$ is completely continuous. Thus we have completed the proof of Lemma 8.

In order to study the existence results for system (3), we consider the following nonlinear operator equation:

$$
x(t)=\lambda \widetilde{\Pi} x(t), \quad \lambda \in(0,1),
$$

where $\widetilde{\Pi}$ is already defined. The following lemma proves that a priori bound exists for the solution of the above equation.

Lemma 9. If the assumptions (H1)-(H10) hold. Then, there exists a priori bound $C^{*}>0$ such that

$$
\left\|x_{t}\right\|_{\mathscr{B}}^{2} \leq C^{*}, \quad t \in J
$$

where $C^{*}$ depends only on $T$ and on the functions $\widehat{\Omega}$ and $\Omega_{i}$, $i=1,2$.

Proof. From (41), by our assumptions, Hölder's inequality and Burkholder-Davis-Gundy's inequality, for $t \in J$, we have

$$
\begin{aligned}
\mathbf{E} \| & x(t) \|^{2} \\
\leq & 14 M_{C}\left[\mathbf{E}\|\varphi(0)\|^{2}+M_{q}\right] \\
& +14 M_{S}\left[\mathbf{E}\left\|x_{1}\right\|^{2}+2\left(M_{g}\|\varphi\|_{\mathscr{B}}^{2}+C_{2}\right)\right] \\
& +14 T^{2} M_{C}\left[M_{g}\left(\left[1+2 M_{\sigma_{1}}\right]\left\|x_{t}\right\|_{\mathscr{B}}^{2}+2 C_{1}\right)+C_{2}\right]+7 T M_{S} \\
& \times \int_{0}^{t} \eta(s) \Omega_{2}\left(\left\|x_{s}\right\|_{\mathscr{B}}^{2}+M_{\sigma} T \int_{0}^{s} \mu(\tau) \Omega_{1}\left(\left\|x_{\tau}\right\|_{\mathscr{B}}^{2}\right) d \tau\right) d s \\
& +14 T^{2} M_{S}\left(M_{h}+T T r(Q) M_{\sigma}\right) \\
& +7 M_{C} \sum_{k=1}^{m} M_{I_{k}^{1}}+7 M_{S} \sum_{k=1}^{m} M_{I_{k}^{2}}
\end{aligned}
$$

Thus, again by Lemma 4, for every $t \in J$, we obtain

$$
\begin{aligned}
& \left\|x_{t}\right\|_{\mathscr{B}}^{2} \\
& \leq 2\|\varphi\|_{\mathscr{B}}^{2}+2 l_{0}^{2} \sup _{s \in[0, t]} \mathrm{E}\|x(s)\|^{2} \leq 2\|\varphi\|_{\mathscr{B}}^{2}+2 l_{0}^{2} \\
& \quad \times\left\{M^{\star}+14 T^{2} M_{C} M_{g}\left[1+2 M_{\sigma_{1}}\right]\left\|x_{t}\right\|_{\mathscr{B}}^{2}+7 T M_{S}\right. \\
& \quad \times \int_{0}^{t} \eta(s) \Omega_{2} \\
& \left.\quad \times\left(\left\|x_{s}\right\|_{\mathscr{B}}^{2}+M_{\sigma} T \int_{0}^{s} \mu(\tau) \Omega_{1}\left(\left\|x_{\tau}\right\|_{\mathscr{B}}^{2}\right) d \tau\right) d s\right\} .
\end{aligned}
$$


Now, we consider the function $\varsigma$ defined by

$$
\varsigma(t):=\sup _{s \in[0, t]}\left\|x_{s}\right\|_{\mathscr{B}}^{2}, \quad t \in J .
$$

Then the function $\varsigma(t)$ is nondecreasing in $J$, and we get

$$
\begin{aligned}
\varsigma(t) \leq & 2\|\varphi\|_{\mathscr{B}}^{2}+2 l_{0}^{2} \sup _{s \in[0, t]} \mathbf{E}\|x(s)\|^{2} \leq 2\|\varphi\|_{\mathscr{B}}^{2}+2 l_{0}^{2} M^{\star} \\
& +28 l_{0}^{2} T^{2} M_{C} M_{g}\left(1+2 M_{\sigma_{1}}\right) \varsigma(t)+14 l_{0}^{2} T M_{S} \\
& \times \int_{0}^{t} \eta(s) \Omega_{2}\left(\varsigma(s)+M_{\sigma} T \int_{0}^{s} \mu(\tau) \Omega_{1}(\varsigma(\tau)) d \tau\right) d s .
\end{aligned}
$$

Consequently,

$$
\begin{aligned}
\varsigma(t) \leq & M_{1}+M_{2} \\
& \times \int_{0}^{t} \eta(s) \Omega_{2}\left(\varsigma(s)+M_{\sigma} T \int_{0}^{s} \mu(\tau) \Omega_{1}(\varsigma(\tau)) d \tau\right) d s .
\end{aligned}
$$

Denoting the right-hand side of the above inequality by $\vartheta(t)$. Then $\vartheta(0)=M_{1}, \varsigma(t) \leq \vartheta(t), t \in J$, and

$$
\vartheta^{\prime}(t) \leq M_{2} \eta(t) \Omega_{2}\left(\varsigma(t)+M_{\sigma} T \int_{0}^{t} \mu(s) \Omega_{1}(\varsigma(s)) d s\right) .
$$

Since $\Omega_{i}, i=1,2$ are nondecreasing, for $t \in J$, we get

$$
\vartheta^{\prime}(t) \leq M_{2} \eta(t) \Omega_{2}\left(\vartheta(t)+M_{\sigma} T \int_{0}^{t} \mu(s) \Omega_{1}(\vartheta(s)) d s\right) .
$$

Let

$$
\rho(t):=\vartheta(t)+M_{\sigma} T \int_{0}^{t} \mu(s) \Omega_{1}(\vartheta(s)) d s .
$$

Then $\rho(0)=\vartheta(0), \vartheta(t) \leq \rho(t)$, and

$$
\begin{aligned}
\rho^{\prime}(t) & \leq \vartheta^{\prime}(t)+M_{\sigma} T \mu(t) \Omega_{1}(\vartheta(t)) \\
& \leq M_{2} \eta(t) \Omega_{2}(\rho(t))+M_{\sigma} T \mu(t) \Omega_{1}(\rho(t)) \\
& \leq \widehat{\Omega}(t)\left(\Omega_{1}(\rho(t))+\Omega_{2}(\rho(t))\right) .
\end{aligned}
$$

This implies that

$$
\begin{aligned}
\int_{\rho(0)}^{\rho(t)} & \frac{d s}{\Omega_{1}(\rho(t))+\Omega_{2}(\rho(t))} \\
& \leq \int_{0}^{T} \widehat{\Omega}(s) d s \leq \int_{M_{1}}^{\infty} \frac{d s}{\Omega_{1}(\rho(t))+\Omega_{2}(\rho(t))},
\end{aligned}
$$

which shows that $\vartheta(t)<\infty$. Thus, there exists a constant $C\left(T, \widehat{\Omega}, \Omega_{1}, \Omega_{2}\right)$ such that $\vartheta(t) \leq C^{*}, t \in J$. So, we get

$$
\left\|x_{t}\right\|_{\mathscr{B}}^{2} \leq \varsigma(t) \leq \vartheta(t) \leq C^{*}, \quad t \in J
$$

Thus we have completed the proof of Lemma 9.
Now, we state the main result of our paper.

Theorem 10. Assume that the assumptions (H1)-(H10) hold. Then, the system (3) has at least one mild solution on J.

Proof. Let us take the set

$$
\begin{aligned}
& \Lambda(\bar{\Pi}) \\
& :=\left\{u \in \mathscr{B}_{T}^{0}: u=\lambda \bar{\Pi}_{1}\left(\frac{u}{x}\right)+\lambda \bar{\Pi}_{2} u \text {, for some } \lambda \in(0,1)\right\} .
\end{aligned}
$$

Then, by Lemma 9 , for any $u \in \Lambda(\bar{\Pi})$, we have $\left\|x_{t}\right\|_{\mathscr{B}}^{2} \leq$ $C^{*}, t \in J$, and hence

$$
\begin{aligned}
\|u\|_{T}^{2} & =\sup _{s \in J}\left(\mathbf{E}\|u(s)\|^{2}\right) \\
& \leq 2 \sup _{s \in J}\left(\mathbf{E}\|x(s)\|^{2}\right)+2 \sup _{s \in J}\left(\mathbf{E}\|\widetilde{\varphi}(s)\|^{2}\right) \\
& \leq 2 \sup _{s \in J} l_{0}^{-2}\left\|x_{t}\right\|_{\mathscr{B}}^{2}+2 \sup _{s \in J} \mathbf{E}\|C(t) \varphi(0)\|^{2} \\
& \leq 2 l_{0}^{-2} C^{*}+2 M_{C} \mathbf{E}\|\varphi(0)\|^{2} .
\end{aligned}
$$

This conclude that $\Lambda$ is bounded on $J$. Consequently, by Krasnoselskii-Schaefer-type fixed point theorem, there exists a fixed point $u^{*}(\cdot)$ for $\bar{\Pi}$ on $B_{r}$ such that $\bar{\Pi} u(t)=u(t)$. Since $x(t)=u^{*}(t)+\widetilde{\varphi}(t), t \in J_{T}, x(\cdot)$ is a fixed point of the operator $\Pi$ which is a mild solution of system (3). The proof for Theorem 10 is thus complete.

Remark 11. In recent years, the stochastic differential equations with Poisson jumps have become very important in modeling the phenomena arising in the fields, such as economics, finance, physics, biology, medicine, and other sciences. It is inspiring that a large number of results about the existence, uniqueness, stability, and invariant measures of stochastic differential equations with Poisson jumps have been reported in the literature. For instance, in [20], Luo and Liu studied the stability of infinite dimensional stochastic evolution with memory and Markovian jumps. Albeverio et al. [21] discussed the existence of global solutions and invariant measures for stochastic differential equations driven by Poisson-type noise with non-Lipschitz coefficients. But there has not been any result on the existence for secondorder impulsive neutral stochastic integrodifferential equations with infinite delays and Poisson jumps. This situation motivates our present research. Therefore, in this remark, we will study the well-posedness for second-order impulsive neutral stochastic integrodifferential equations with nonlocal conditions, infinite delays, and Poisson jumps in the form

$$
\begin{aligned}
d\left[x^{\prime}(t)\right. & \left.-g\left(t, x_{t}, \int_{0}^{t} \sigma_{1}\left(t, s, x_{s}\right) d s\right)\right] \\
= & {\left[A x(t)+f\left(t, x_{t}, \int_{0}^{t} \sigma_{2}\left(t, s, x_{s}\right) d s\right)\right] d t } \\
& +\int_{-\infty}^{t} \sigma\left(t, s, x_{s}\right) d W(s) \\
& +\int_{\mathscr{U}} \gamma(t, x(t-), v) \widetilde{N}(d t, d v), \quad t_{k} \neq t \in J:=[0, T],
\end{aligned}
$$




$$
\begin{gathered}
\Delta x\left(t_{k}\right)=I_{k}^{1}\left(x\left(t_{k}^{-}\right)\right), \quad k=\{1, \ldots, m\}=: \overline{1, m}, \\
\Delta x^{\prime}\left(t_{k}\right)=I_{k}^{2}\left(x\left(t_{k}^{-}\right)\right), \quad k=\overline{1, m}, \\
x^{\prime}(0)=x_{1} \in \mathbb{H}, \\
x(0)-q\left(x_{t_{1}}, x_{t_{2}}, \ldots, x_{t_{n}}\right)=x_{0}=\varphi \in \mathscr{B}, \\
\text { for a.e. } s \in J_{0}:=(-\infty, 0],
\end{gathered}
$$

where the functions $g, f, \sigma_{1}, \sigma_{2}, \sigma, q$, and $I_{k}^{1}, I_{k}^{2}$ are defined as in Theorem 10; $\gamma: J \times \mathbb{H} \times \mathscr{U} \rightarrow \mathbb{H}$ are appropriate mappings which will be specified later; $\widetilde{N}(d s, d v)$ is a compensated Poisson random measure induced by Poisson point process $p(\cdot)$, which is independent of the Wiener process $W$ and takes values in a measurable space $(\mathcal{U}, \mathfrak{B}(\mathcal{U}))$ with a $\sigma$-finite intensity measure $\lambda(d v)$ by $N(d t, d v)$ the Poisson counting measure associated with $p(\cdot)$; that is, $N(t, \mathcal{U})=\sum_{s \in D_{p}, s \leq t} \mathbb{\square}_{\mathcal{U}}(p(s))$, for any measurable set $\mathcal{U} \in \mathfrak{B}(\mathbb{K}-\{0\})$, which denotes the Borel $\sigma$-field of $(\mathbb{K}-\{0\})$. Let

$$
\widetilde{N}(d t, d v):=N(d t, d v)-\lambda(d v) d t
$$

Denote by $\mathscr{P}^{2}(J \times \mathscr{U} ; \sharp)$ the space of all predictable mappings $\gamma: J \times \mathscr{U} \rightarrow \mathbb{H}$ for which

$$
\int_{0}^{t} \int_{\mathscr{U}} \mathbf{E}\|\gamma(t, v)\|_{\mathbb{Q}}^{2} \lambda(d v) d t<\infty .
$$

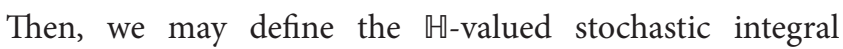
$\int_{0}^{t} \int_{\mathscr{U}} \gamma(t, v) \widetilde{N}(d t, d v)$, which is a centred square-integrable martingale. For the construction of this kind of integral, we refer the reader to Protter [22].

Let $\varphi(t) \in \mathscr{L}_{2}(\Omega, \mathscr{B})$ and let $x_{1}(t)$ be $\mathbb{H}$-valued $\mathscr{F}_{t^{-}}$ measurable random variable independent of the Wiener process $W$ and the Poisson point process $p(\cdot)$, with finite second moment.

Next, we give the definition of mild solution for (11).

Definition 12. An $\mathscr{F}_{t}$-adapted càdlàg stochastic process $x$ : $J_{T} \rightarrow \mathbb{H}$ is called a mild solution of (3) on $J_{T}$ if $x(0)-$ $q\left(x_{t_{1}}, x_{t_{2}}, \ldots, x_{t_{n}}\right)=x_{0}=\varphi \in \mathscr{B}$ satisfying $q, x_{0} \in$ $\mathscr{L}_{2}^{0}(\Omega, \mathbb{H})$ and $x^{\prime}(0)=x_{1} \in \mathbb{H}$ satisfying $x_{1} \in \mathscr{L}_{2}^{0}(\Omega, \mathbb{H})$; the functions $C(t-s) g\left(s, x_{s}, \int_{0}^{s} \sigma_{1}\left(s, \tau, x_{\tau}\right) d \tau\right)$ and $S(t-$ $s) f\left(s, x_{s}, \int_{0}^{s} \sigma_{2}\left(s, \tau, x_{\tau}\right) d \tau\right)$ are integrable on $J$ such that the following conditions hold:

(i) $\left\{x_{t}: t \in J\right\}$ is a $\mathscr{B}$-valued stochastic process;

(ii) for arbitrary $t \in J, x(t)$ satisfies the following integral equation:

$$
\begin{aligned}
x(t)= & C(t)\left[\varphi(0)+q\left(x_{t_{1}}, x_{t_{2}}, \ldots, x_{t_{n}}\right)(0)\right] \\
& +S(t)\left[x_{1}-g\left(0, x_{0}, 0\right)\right] \\
& +\int_{0}^{t} C(t-s) g\left(s, x_{s}, \int_{0}^{s} \sigma_{1}\left(s, \tau, x_{\tau}\right) d \tau\right) d s
\end{aligned}
$$

$$
\begin{aligned}
& +\int_{0}^{t} S(t-s) f\left(s, x_{s}, \int_{0}^{s} \sigma_{2}\left(s, \tau, x_{\tau}\right) d \tau\right) d s \\
& +\sum_{0<t_{k}<t} C\left(t-t_{k}\right) I_{k}^{1}\left(x\left(t_{k}^{-}\right)\right) \\
& +\int_{0}^{t} S(t-s) \int_{-\infty}^{s} \sigma\left(s, \tau, x_{\tau}\right) d W(\tau) d s \\
& +\sum_{0<t_{k}<t} S\left(t-t_{k}\right) I_{k}^{2}\left(x\left(t_{k}^{-}\right)\right) \\
& +\int_{0}^{t} S(t-s) \int_{\mathscr{U}} \gamma(t, x(t-), v) \widetilde{N}(d t, d v) ;
\end{aligned}
$$

(iii) $\Delta x\left(t_{k}\right)=I_{k}^{1}\left(x\left(t_{k}^{-}\right)\right), \Delta x^{\prime}\left(t_{k}\right)=I_{k}^{2}\left(x\left(t_{k}^{-}\right)\right), k=\overline{1, m}$.

To establish the well-posedness of mild solution for second-order impulsive neutral stochastic integrodifferential equations with nonlocal conditions, infinite delays, and Poisson jumps, we need the following assumption.

(H11) For any $x, y \in \mathbb{H}$, and $t \in J$ such that

$$
\begin{aligned}
& \int_{\mathscr{U}}\|\gamma(t, x(s-), v)-\gamma(t, y(s-), v)\|_{\mathbb{Q}}^{2} \lambda(d v) \\
& \quad \vee\left(\int_{\mathscr{U}^{-}}\left\|\gamma\left(t, x\left(s^{-}\right), v\right)-\gamma\left(t, y\left(s^{-}\right), v\right)\right\|_{\mathbb{Q}}^{4} \lambda(d v)\right)^{1 / 2} \\
& \quad \leq M_{\gamma}\|x(s)-y(s)\|_{\mathbb{a}}^{2},
\end{aligned}
$$

where $M_{\gamma}$ is a positive constant.

Theorem 13. Assume that the assumptions (H1)-(H11) hold. If

$$
\begin{gathered}
4 l_{0}^{2} T M_{C} M_{g}\left(1+M_{\sigma_{1}}\right)+2 T C M_{\gamma}<1 \\
C=C(T) \text { is a positive constant, }
\end{gathered}
$$

then the system (11) has at least one mild solution on J.

Proof. By adapting and employing the techniques used in Theorem 10, we can easily prove the conclusion of Theorem 13. Indeed, similar to the above discussion, we consider the mapping $\bar{\Pi}: \mathscr{B}_{T}^{0} \rightarrow \mathscr{B}_{T}^{0}$ defined by $\bar{\Pi} u(t)=0$, for $t \in J_{0}$ and

$$
\begin{aligned}
& \bar{\Pi} u(t) \\
& =S(t)\left[u_{1}-g\left(0, \widetilde{\varphi}_{0}, 0\right)\right] \\
& \quad+\int_{0}^{t} C(t-s) g\left(s, u_{s}+\widetilde{\varphi}_{s}, \int_{0}^{s} \sigma_{1}\left(s, \tau, u_{\tau}+\widetilde{\varphi}_{\tau}\right) d \tau\right) d s \\
& \quad+\int_{0}^{t} S(t-s) f\left(s, u_{s}+\widetilde{\varphi}_{s}, \int_{0}^{s} \sigma_{2}\left(s, \tau, u_{\tau}+\widetilde{\varphi}_{\tau}\right) d \tau\right) d s
\end{aligned}
$$




$$
\begin{aligned}
& +\int_{0}^{t} S(t-s)\left[h(s)+\int_{0}^{s} \sigma\left(s, \tau, u_{\tau}+\widetilde{\varphi}_{\tau}\right) d W(\tau)\right] d s \\
& +\sum_{0<t_{k}<t} C\left(t-t_{k}\right) I_{k}^{1}\left(u\left(t_{k}^{-}\right)+\widetilde{\varphi}\left(t_{k}^{-}\right)\right) \\
& +\sum_{0<t_{k}<t} S\left(t-t_{k}\right) I_{k}^{2}\left(u\left(t_{k}^{-}\right)+\widetilde{\varphi}\left(t_{k}^{-}\right)\right) \\
& +\int_{0}^{t} S(t-s) \int_{\mathcal{U}} \gamma(t, u(t-)+\widetilde{\varphi}(t-), v) \widetilde{N}(d t, d v),
\end{aligned}
$$

$t \in J$.

Now, for $t \in J$, we decompose $\bar{\Pi}$ as $\bar{\Pi}=\bar{\Pi}_{1}+\bar{\Pi}_{2}$, where

$$
\begin{aligned}
& \bar{\Pi}_{1} u(t) \\
& =S(t)\left[u_{1}-g\left(0, \widetilde{\varphi}_{0}, 0\right)\right] \\
& \quad+\int_{0}^{t} C(t-s) g\left(s, u_{s}+\widetilde{\varphi}_{s}, \int_{0}^{s} \sigma_{1}\left(s, \tau, u_{\tau}+\widetilde{\varphi}_{\tau}\right) d \tau\right) d s \\
& \quad+\int_{0}^{t} S(t-s) \int_{\mathcal{U}} \gamma(t, u(t-)+\widetilde{\varphi}(t-), v) \widetilde{N}(d t, d v), \\
& \bar{\Pi}_{2} u(t) \\
& =\int_{0}^{t} S(t-s) f\left(s, u_{s}+\widetilde{\varphi}_{s}, \int_{0}^{s} \sigma_{2}\left(s, \tau, u_{\tau}+\widetilde{\varphi}_{\tau}\right) d \tau\right) d s \\
& \quad+\int_{0}^{t} S(t-s)\left[h(s)+\int_{0}^{s} \sigma\left(s, \tau, u_{\tau}+\widetilde{\varphi}_{\tau}\right) d W(\tau)\right] d s \\
& \quad+\sum_{0<t_{k}<t} C\left(t-t_{k}\right) I_{k}^{1}\left(u\left(t_{k}^{-}\right)+\widetilde{\varphi}\left(t_{k}^{-}\right)\right) \\
& \quad+\sum_{0<t_{k}<t} S\left(t-t_{k}\right) I_{k}^{2}\left(u\left(t_{k}^{-}\right)+\widetilde{\varphi}\left(t_{k}^{-}\right)\right) .
\end{aligned}
$$

Next, we will show that the operators $\bar{\Pi}_{1}, \bar{\Pi}_{2}$ satisfy all the conditions of Lemma 5. Lemma 8 has shown that the operator $\bar{\Pi}_{2}$ is completely continuous. Moreover, by assumptions (H1)-(H3), (H11), Lemma 4, the BurkholderDavis-Gundy inequality for pure jump stochastic integral in Hilbert space (Lemma 2.2 in [20]), and assumption (61), for each $t \in J$, we conclude immediately that $\bar{\Pi}_{1}$ is contractive.

Finally, by using the same arguments as Theorem 10, we infer that there exists a mild solution of the system (11). This completes the proof of Theorem 13.

\section{Application}

As we know, wave equations subject to random excitations have been intensively studied in the last forty years for their applications in physics, relativistic quantum mechanics, or oceanography; see, for instance, [23-27] and the references therein. The stochastic wave equation is one of the fundamental stochastic partial differential equations of hyperbolic type. The well-posedness of its solutions is significantly different from those of solutions to other stochastic partial differential equations, such as the stochastic heat equation or the stochastic Laplace equation. Therefore, in this section, an example on the stochastic nonlinear wave equation will be provided to illustrate the obtained theory. Specifically, we consider the existence of the following impulsive neutral stochastic nonlinear wave equations with nonlocal conditions and infinite delays of the form

$$
\begin{gathered}
\frac{\partial}{\partial t}\left[\frac{\partial}{\partial t} y(t, \xi)-\int_{-\infty}^{t} \delta_{1}(t, \xi, s-t,) P_{1}(y(s, \xi)) d s\right. \\
\left.-\int_{0}^{t} \int_{-\infty}^{s} b_{1}(s-\tau) P_{2}(y(\tau, \xi)) d \tau d s\right] \\
=\left[\frac{\partial^{2}}{\partial \xi^{2}} y(t, \xi)+\int_{-\infty}^{t} \delta_{2}(t, \xi, s-t,) G_{1}(y(s, \xi)) d s\right. \\
\left.+\int_{0}^{t} \int_{-\infty}^{s} b_{2}(s-\tau) G_{2}(y(\tau, \xi)) d \tau d s\right] d t \\
+\int_{-\infty}^{t} \delta(s-t) y(t, \xi) d \beta(s), \\
t \neq t_{k}, t \in J, \xi \in[0, \pi], \\
\Delta y\left(t_{k}\right)(\xi)=\int_{-\infty}^{t_{k}} \eta_{k}\left(t_{k}-s\right) y(s, \xi) d s \\
k=\overline{1, m}, \xi \in[0, \pi], \\
\Delta y^{\prime}\left(t_{k}\right)(\xi)=\int_{-\infty}^{t_{k}} \rho_{k}\left(t_{k}-s\right) y(s, \xi) d s, \\
k=\overline{1, m}, \xi \in[0, \pi], \\
y(t, 0)=y(t, \pi)=0, \quad t \in J \\
\frac{\partial}{\partial t} y(0, \xi)=x_{1}(\xi), \\
y(t, \xi)-\sum_{i=1}^{n} \int_{0}^{\pi} p_{i}(\xi, \zeta) y\left(t_{i}, \zeta\right) d \zeta=\varphi(t, \xi), \\
t \in J_{0}, \xi \in[0, \pi],
\end{gathered}
$$

where $\beta(t)$ is a standard one-dimensional Wiener process in $\mathbb{W}$ defined on a stochastic basis $(\Omega, \mathscr{F}, \mathbf{P}), 0<t_{1}<t_{2}<\cdots<$ $t_{n}<T, n \in \mathbb{N}, 0=t_{0}<t_{1}<\cdots<t_{m}<T$ are prefixed numbers, and $\varphi \in \mathscr{B}$. We take $\mathbb{\sharp}=L^{2}([0, \pi])$ with the norm $\|\cdot\|$. Define $A: \mathbb{\sharp} \rightarrow \mathbb{U}$ by $A x=x^{\prime \prime}$ with domain

$$
\begin{gathered}
D(A)=\left\{x(\cdot) \in \mathbb{U}: x, x^{\prime}\right. \text { are absolutely continuous, } \\
\left.x^{\prime \prime} \in \mathbb{U}, x(0)=x(\pi)=0\right\} .
\end{gathered}
$$

The spectrum of $A$ consists of the eigenvalues $-n^{2}$ for $n \in$ $\mathbb{N}$, with associated eigenvectors $e_{n}(\xi):=\sqrt{2 / \pi} \sin n \xi, n=$ $1,2,3, \ldots$. Furthermore, the set $\left\{e_{n}: n \in \mathbb{N}\right\}$ is an orthogonal basics in $\mathbb{H}$. Then

$$
A x=\sum_{n=1}^{\infty} n^{2}\left\langle x, e_{n}\right\rangle e_{n}, \quad x \in D(A) .
$$


Using (66), one can easily verify that the operators $C(t)$ defined by

$$
C(t) x=\sum_{n=1}^{\infty} \cos (n t)\left\langle x, e_{n}\right\rangle e_{n}, \quad t \in \mathbb{R},
$$

form a cosine function on $\mathbb{H}$, with associated sine function

$$
S(t) x=\sum_{n=1}^{\infty} \frac{\sin (n t)}{n}\left\langle x, e_{n}\right\rangle e_{n}, \quad t \in \mathbb{R} .
$$

It is clear that (see [17]) for all $x \in \mathbb{Z}, t \in \mathbb{R}, C(\cdot) x$ and $S(\cdot) x$ are periodic functions with $\|C(t)\| \leq 1$ and $\|S(t)\| \leq 1$. Thus, $(\mathrm{H} 1)$ is true.

Now, we give a special $\mathscr{B}$-space. Let $l(s)=e^{2 s}, s \leq 0$; then $l_{0}=\int_{J_{0}} l(s) d s=1 / 2$ and define

$$
\|\psi\|_{\mathscr{B}}=\int_{J_{0}} l(s) \sup _{\theta \in[s, 0]}\left(\mathbf{E}\|\psi(\theta)\|^{2}\right)^{1 / 2} d s, \quad \forall \psi \in \mathscr{B} .
$$

It follows from [18] that $\left(\mathscr{B},\|\cdot\|_{\mathscr{B}}\right)$ is a Banach space. Hence for $(t, \psi) \in J \times \mathscr{B}$, where $\psi(\theta) x=\psi(\theta, x),(\theta, x) \in J_{0} \times[0, \pi]$. Let $y(t)(\xi)=y(t, \xi)$.

To study the system (64), we assume that the following conditions hold.

(i) The functions $p_{i}:[0, \pi] \times[0, \pi] \rightarrow \mathbb{R}$ are $C^{2}-$ functions, for each $i=\overline{1, n}$.

(ii) The functions $\eta_{k}, \rho_{k} \in \mathrm{C}(\mathbb{R}, \mathbb{R})$ such that for $k=\overline{1, m}$,

$$
\begin{aligned}
& \bar{M}_{I_{k}^{1}}=\int_{J_{0}} l(s) \eta_{k}^{2}(s) d s<\infty, \\
& \bar{M}_{I_{k}^{2}}=\int_{J_{0}} l(s) \rho_{k}^{2}(s) d s<\infty .
\end{aligned}
$$

If we put

$$
\begin{aligned}
& g\left(t, \psi, V_{1} \psi\right)(\xi)= \int_{J_{0}} \delta_{1}(t, \xi, \theta) P_{1}(\psi(\theta)(\xi)) d \theta \\
&+V_{1} \psi(\xi), \\
& f\left(t, \psi, V_{2} \psi\right)(\xi)= \int_{J_{0}} \delta_{2}(t, \xi, \theta) G_{1}(\psi(\theta)(\xi)) d \theta \\
&+V_{2} \psi(\xi), \\
& \sigma(t, s, \psi)(\xi)=\int_{J_{0}} \delta(\theta) \psi(\theta)(\xi) d \theta, \\
& I_{k}^{1}(t, \psi)(\xi)=\int_{J_{0}} \eta_{k}(-s) \psi(\theta)(\xi) d s, \quad k=\overline{1, m}, \\
& I_{k}^{2}(t, \psi)(\xi)=\int_{J_{0}} \rho_{k}(-s) \psi(\theta)(\xi) d s, \quad k=\overline{1, m},
\end{aligned}
$$

where

$$
\begin{aligned}
& V_{1} \psi(\xi)=\int_{0}^{t} \int_{J_{0}} b_{1}(s-\theta) P_{2}(\psi(\theta)(\xi)) d \theta d s \\
& V_{2} \psi(\xi)=\int_{0}^{t} \int_{J_{0}} b_{2}(s-\theta) G_{2}(\psi(\theta)(\xi)) d \theta d s
\end{aligned}
$$

Then, the system (64) can be written in the abstract form as the system (3). Further, we can impose some suitable conditions on the above defined functions as those in the assumptions (H1)-(H10). Therefore, by Theorem 10, we can conclude that the system (64) has a mild solution on $J$.

\section{Conclusion}

In this paper, we have discussed the existence for a class of second-order impulsive neutral stochastic integrodifferential equations with nonlocal conditions and infinite delays in a Hilbert space. By using the Krasnoselskii-Schaefer-type fixed point theorem combined with theories of a strongly continuous cosine family of bounded linear operators, the wellposedness of mild solution for the second-order impulsive neutral stochastic integrodifferential equations with nonlocal conditions and infinite delays is obtained. Besides, if the system (3) is added to the Poisson jumps, then we also get the existence of mild solution for second-order impulsive neutral stochastic integrodifferential equations with nonlocal conditions, infinite delays, and Poisson jumps. Finally, an example illustrating the applicability of the general theory is also provided.

\section{Conflict of Interests}

The author declares that there is no conflict of interests regarding the publication of this paper.

\section{References}

[1] V. Lakshmikantham, D. Bainov, and P. S. Simeonov, Theory of Impulsive Differential Equations, vol. 6 of Series in Modern Applied Mathematics, World Scientific Publishing, Teaneck, NJ, USA, 1989.

[2] Y.-K. Chang, "Controllability of impulsive functional differential systems with infinite delay in Banach spaces," Chaos, Solitons \& Fractals, vol. 33, no. 5, pp. 1601-1609, 2007.

[3] Y.-K. Chang, A. Anguraj, and M. M. Arjunan, "Existence results for impulsive neutral functional differential equations with infinite delay," Nonlinear Analysis: Hybrid Systems, vol. 2, no. 1, pp. 209-218, 2008.

[4] E. Hernández Morales, "A second-order impulsive cauchy problem," International Journal of Mathematics and Mathematical Sciences, vol. 31, no. 8, pp. 451-461, 2002.

[5] J. Y. Park, K. Balachandran, and N. Annapoorani, "Existence results for impulsive neutral functional integrodifferential equations with infinite delay," Nonlinear Analysis: Theory, Methods \& Applications, vol. 71, no. 7-8, pp. 3152-3162, 2009.

[6] G. Cao, Stochastic differential evolution equations in infinite dimensional [M.S. thesis], Huazhong University of Science and Technology, 2005.

[7] R. Sakthivel and J. Luo, "Asymptotic stability of nonlinear impulsive stochastic differential equations," Statistics and Probability Letters, vol. 79, no. 9, pp. 1219-1223, 2009.

[8] Z. M. Yan and X. X. Yan, "Existence of solutions for impulsive partial stochastic neutral integrodif- ferential equations with state-dependent delay," Collectanea Mathematica, vol. 64, no. 2, pp. 235-250, 2013. 
[9] J. Cui and L. Yan, "Existence result for fractional neutral stochastic integro-differential equations with infinite delay," Journal of Physics A, vol. 44, no. 33, Article ID 335201, 16 pages, 2011.

[10] G. Arthi and K. Balachandran, "Controllability of damped second-order impulsive neutral functional differential systems with infinite delay," Journal of Optimization Theory and Applications, vol. 152, no. 3, pp. 799-813, 2012.

[11] P. Balasubramaniam and P. Muthukumar, "Approximate controllability of second-order stochastic distributed implicit functional differential systems with infinite delay," Journal of Optimization Theory and Applications, vol. 143, no. 2, pp. 225-244, 2009.

[12] J. Cui and L. Yan, "Existence results for impulsive neutral second-order stochastic evolution equations with nonlocal conditions," Mathematical and Computer Modelling, vol. 57, no. 9-10, pp. 2378-2387, 2013.

[13] N. I. Mahmudov and M. A. McKibben, "Abstract secondorder damped McKean-Vlasov stochastic evolution equations," Stochastic Analysis and Applications, vol. 24, no. 2, pp. 303-328, 2006.

[14] G. da Prato and J. Zabczyk, Stochastic Equations in Infinite Dimensions, vol. 44, Cambridge University Press, Cambridge, UK, 1992.

[15] H. O. Fattorini, Second Order Linear Differential Equations in Banach Spaces, vol. 108 of North-Holland Mathematics Studies, North-Holland, Amsterdam, The Netherlands, 1985.

[16] C. C. Travis and G. F. Webb, "Cosine families and abstract nonlinear second order differential equations," Acta Mathematica Academiae Scientiarum Hungaricae, vol. 32, no. 1-2, pp. 75-96, 1978.

[17] C. C. Travis and G. F. Webb, "Compactness, regularity, and uniform continuity properties of strongly continuous cosine families," Houston Journal of Mathematics, vol. 3, no. 4, pp. 555567, 1977.

[18] Y. Hino, S. Murakami, and T. Naito, Functional Differential Equations with Infinite Delay, vol. 1473 of Lecture Notes in Mathematics, Springer, Berlin, Germany, 1991.

[19] T. A. Burton and C. Kirk, "A fixed point theorem of Krasnoselskii-Schaefer type," Mathematische Nachrichten, vol. 189, no. 1, pp. 23-31, 1998.

[20] J. Luo and K. Liu, "Stability of infinite dimensional stochastic evolution equations with memory and Markovian jumps," Stochastic Processes and Their Applications, vol. 118, no. 5, pp. 864-895, 2008.

[21] S. Albeverio, Z. Brzeźniak, and J.-L. Wu, "Existence of global solutions and invariant measures for stochastic differential equations driven by Poisson type noise with non-Lipschitz coefficients," Journal of Mathematical Analysis and Applications, vol. 371, no. 1, pp. 309-322, 2010.

[22] P. E. Protter, Stochastic Integration and Differential Equations, Springer, New York, NY, USA, 2nd edition, 2004.

[23] P.-L. Chow, "Stochastic wave equations with polynomial nonlinearity," Annals of Applied Probability, vol. 12, no. 1, pp. 361-381, 2002.

[24] R. C. Dalang, "Extending martingale measure stochastic integral with applications to spatially homogeneous S.P.D.E's," Electronic Journal of Probability, vol. 4, no. 6, pp. 1-29, 1999.
[25] R. C. Dalang and O. Lévêque, "Second-order linear hyperbolic SPDEs driven by isotropic gaussian noise on a sphere," Annals of Probability, vol. 32, no. 1B, pp. 1068-1099, 2004.

[26] S. Peszat and J. Zabczyk, "Nonlinear stochastic wave and heat equations," Probability Theory and Related Fields, vol. 116, no. 3, pp. 421-443, 2000.

[27] J. B. Walsh, "An introduction to stochastic partial differential equations," in École d'Été de Probabilités de Saint Flour XIV1984, vol. 1180 of Lecture Notes in Mathematics, pp. 265-439, Springer, Berlin, Germany, 1986. 


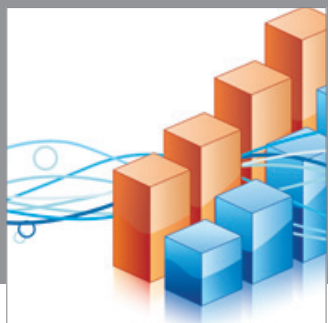

Advances in

Operations Research

mansans

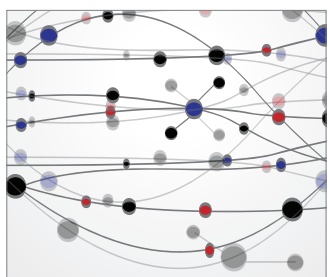

The Scientific World Journal
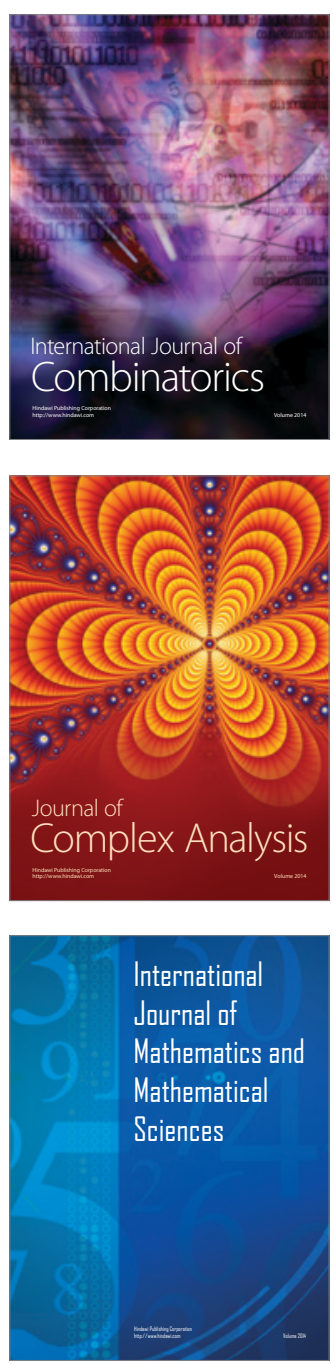
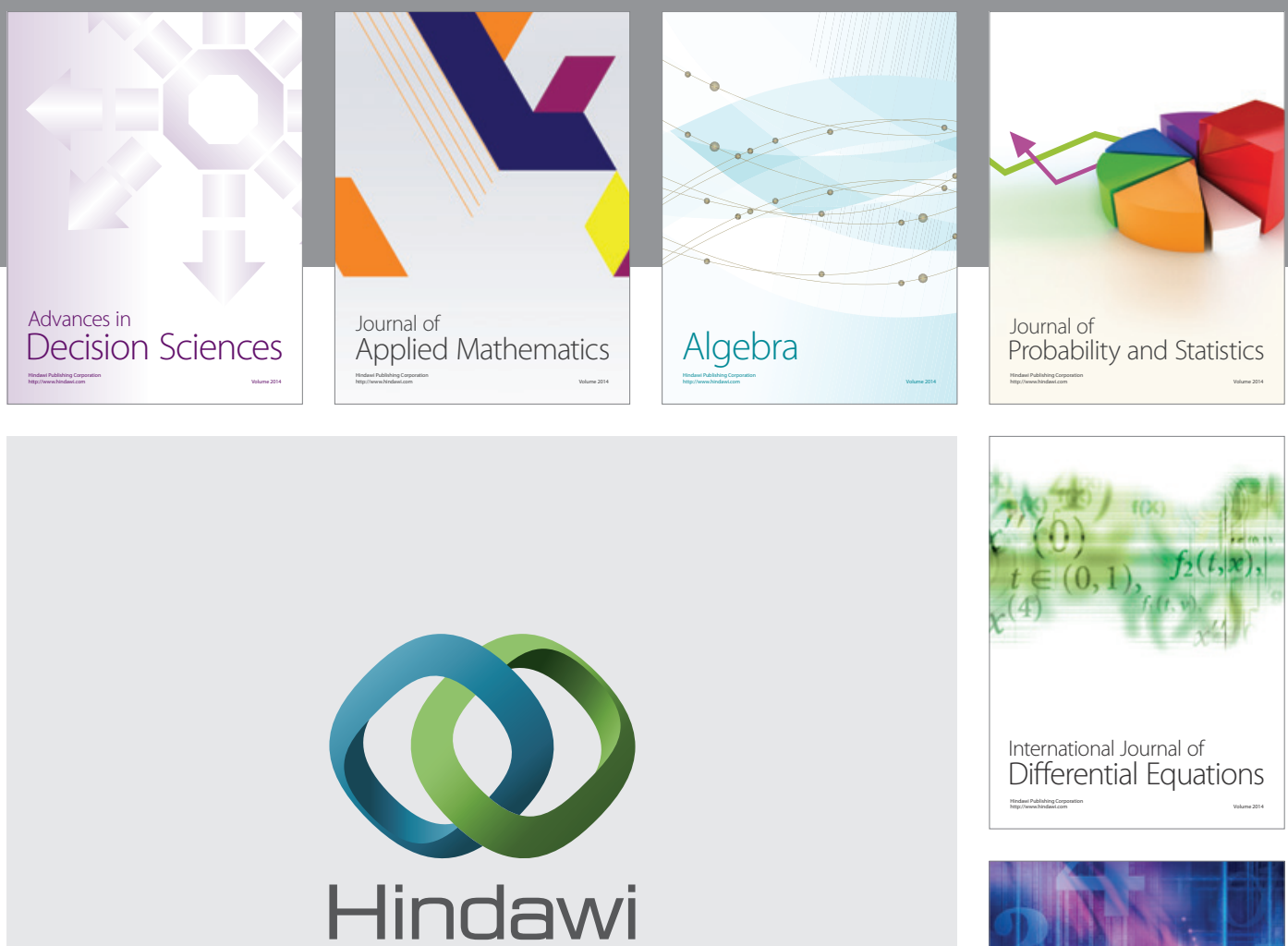

Submit your manuscripts at http://www.hindawi.com
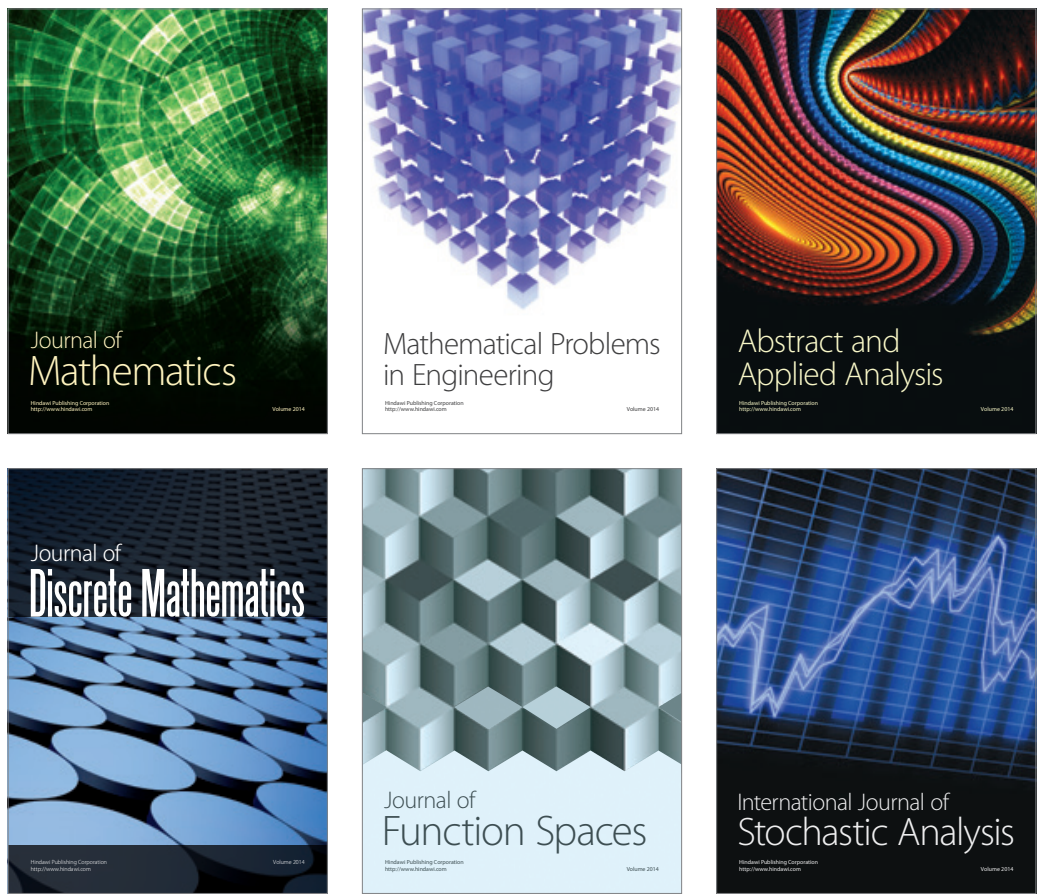

Journal of

Function Spaces

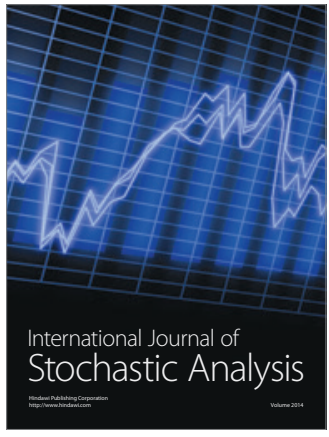

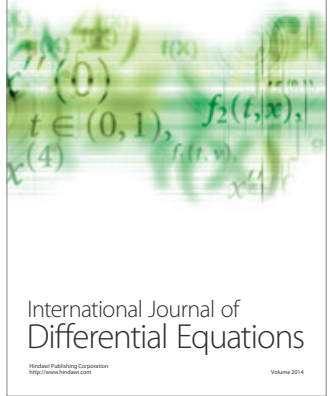
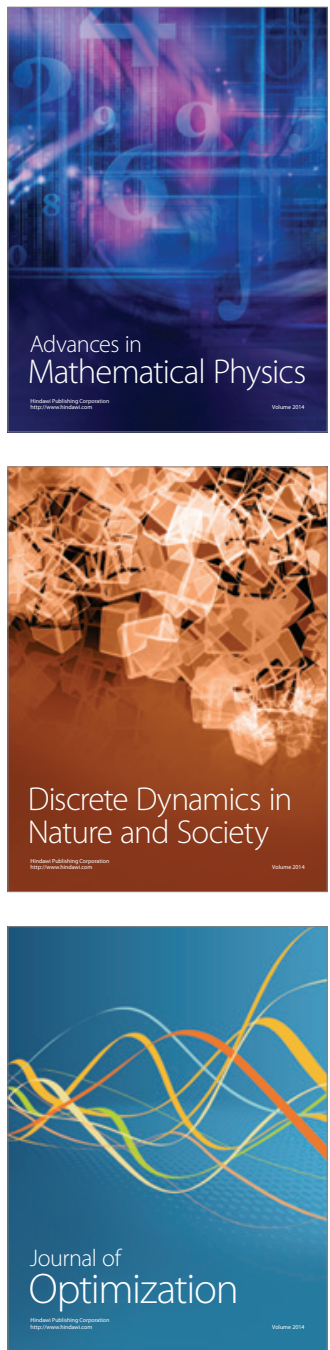